\title{
Colloidal Characterization and Thermodynamic Stability of Binary Eye Lens Protein Mixtures
}

\author{
N. Dorsaz, ${ }^{\dagger}$ G. M. Thurston,,$\stackrel{\perp}{\ddagger}$ A. Stradner, ${ }^{\S}$ P. Schurtenberger, ${ }^{\S, \|}$ and G. Foffi $*, \uparrow$ \\ Institut Romand de Recherche Numérique en Physique des Matériaux, (IRRMA) and Institute of Theoretical \\ Physics (ITP), Ecole Polytechnique Fédérale de Lausanne (EPFL), CH-1015 Lausanne, Switzerland, \\ Department of Physics, Rochester Institute of Technology, Rochester, New York 14623-5603, and Adolphe \\ Merkle Institute and Fribourg Center for Nanomaterials, University of Fribourg, CH-1700 Fribourg, \\ Switzerland
}

We present a study of binary mixtures of eye lens crystallin proteins. A coarse-grained model of aqueous $\alpha$ and $\gamma$-crystallin mixtures based on molecular dynamics simulations and SANS experiments is proposed. Thermodynamic perturbation theory is implemented to obtain the stability boundaries, or spinodal surface, of the binary mixture in the full parameter space. The stability of these high-concentration crystallin mixtures was found to depend on the $\alpha-\gamma$ attraction in a manner that is both extremely sensitive and nonmonotonic; stronger or weaker attraction resulted in a spectacularly enhanced instability. The relevance of these mechanisms as possible sources of the alteration of the spatial distribution of the lens proteins encountered in cataract disease is discussed.

\section{Introduction}

Proteins are attracting growing attention within the colloid physics community due to their suitability as model colloids, and this possibility of exploiting proteins as ideal colloids has already given promising results. Solutions of lysozyme, the physicist's protein, were found to exhibit a metastable liquid-liquid phase separation and possess a cluster phase at low ionic strength, and evidence for a glass line was also discovered. ${ }^{1-3}$ The advantages of proteins are clear. In terms of structure, the often perfect monodispersity of single-chain proteins is a property currently out of reach for synthesis of organic and inorganic colloidal particles. Interprotein interactions also encompass richer and more interesting scenarios than are usually encountered in atomic systems. In fact, since proteins carry $\mathrm{pH}$-dependent surface charges, their electrostatic interaction can be easily tuned by modifying the solvent properties ( $\mathrm{pH}$, ionic strength). Moreover, several proteins exhibit shortrange attractive interactions whose strength can be modulated by simply varying the temperature. ${ }^{4}$ All of these features make proteins an essential playground for understanding the equilibrium and nonequilibrium phase behavior of colloidal systems in general. As a matter of fact, the reverse is also true, and both colloidal and statistical physics are giving important contributions to the understanding of protein behavior, especially in phenomena like aggregation, phase transition, or dynamical arrest. These are all phenomena that involve a large number of proteins and for which atomistic approaches are clearly not feasible.

In analyzing colloidal systems, a coarse-graining procedure, which consists of integrating out a subset of the degrees of freedom, is usually the first and unavoidable step. This procedure

\footnotetext{
* Towhom correspondence shouldbeaddressed.E-mail: giuseppe.foffi@epfl.ch

Ecole Polytechnique Fédérale de Lausanne (EPFL).

* Rochester Institute of Technology.

$\S$ University of Fribourg.

"E-mail: peter.schurtenberger@unifr.ch.

${ }^{\perp}$ E-mail: george.thurston@rit.edu.
}

is required to reduce the complexity of the interactions between components (electrostatic, hydrophobic, steric, entropic, etc.) to an effective potential. ${ }^{5,6}$ Once a suitable coarse-grained interaction has been devised, one can use the tools of statistical mechanics for simple liquids ${ }^{7}$ to study the system. In practice, colloidal particles are treated as "big atoms" 8,9 with specific interaction potentials. In this way, insights have been obtained in a variety of contexts, including colloids, colloid-polymers systems, gels, glasses, and aggregation kinetics (for recent reviews, see, for example, refs 5, 10, and 11). Moreover, this approach has been successfully extended to protein solutions and has proven to be extremely valuable also for these more complicated systems. ${ }^{12,13}$

The colloidal models emerging from this interdisciplinary research are also giving new insights into numerous questions of biological and medical relevance. A broad and constantly growing class of diseases, such as Alzheimer's disease, sicklecell disease, and cataract disease, to name only a few, involve protein association phenomena as an essential aspect. The basic element common to all members of this class of molecular condensation diseases ${ }^{14}$ is an attractive energy of interaction between specific biologic molecules which produces condensation into dense, frequently insoluble mesoscopic phases. It appears that it is the subtle interplay between interprotein attraction and repulsion that leads to the condensed protein phase. Among this class of diseases, cataract is particularly important because it is the world's leading cause of blindness, and effective prevention or nonsurgical cure is still lacking. ${ }^{15}$ This disease is most often the consequence of an uncontrolled aggregation (or phase separation) of the proteins in the eye lens that results in a loss of its transparency.

The cells of mammalian eye lenses contain an aqueous solution of highly concentrated lens-specific proteins and are normally sufficiently transparent for vision. To understand the origin of normal lens transparency, it is essential to recognize that each of the proteins in the lens does not scatter light independently of its neighbors. Instead, the scattering in the eye 
lens is reduced by the correlation in the position of pairs of proteins. ${ }^{16}$ In a normal lens, the local fluctuations of the concentration of proteins, on length scales comparable to the light wavelength, are small.

One of the principal ways of disrupting the needed local homogeneity in the eye lens is by the aggregation of lens proteins into high-molecular-weight clusters that produce sufficient fluctuations to enhance the turbidity and cause cataract. The needed homogeneous packing of lens proteins has indeed long been recognized to be fundamental for eye lens transparency and has been characterized at molecular length scales using small-angle X-ray scattering. ${ }^{17}$ Many studies of this nature make cold cataract an example of a protein condensation disease where concepts from colloid science have already been successfully applied.

The highly packed proteins in the eye lens fiber cells are named "crystallins," and the most common mammalian crystallins belong to the $\alpha, \beta$, and $\gamma$ families. In this study, we have focused on the $\alpha$ - and $\gamma$-crystallins. The former are globular, polydisperse, multisubunit, $800 \mathrm{kDa}$ proteins, whose interactions can be described to a good approximation with a simple hardsphere colloid model. ${ }^{18}$ The $\gamma$-crystallins are monomeric, with a molecular weight of about $21 \mathrm{kDa}$. The discovery of a metastable liquid-liquid phase separation provided the evidence for a short-range attraction between $\gamma$-crystallins, and the use of the corresponding colloid model has led to a quantitative description of the phase behavior. ${ }^{19-24}$

Crystallin aggregation and liquid-liquid phase separation can both produce density inhomogeneities that are principal sources of increased light scatter in cataract and therefore continue to be major themes of lens protein research. Liquid-liquid phase separation not only gives rise to the so-called cold cataract ${ }^{25}$ but also dramatically enhances light scattering at body temperature, well above the critical point. ${ }^{22,26-28}$ The proximity of liquid-liquid phase separation also substantially affects the thermodynamic properties of lens protein mixtures ${ }^{28-31}$ and is therefore an important aspect of understanding the driving forces for chemical change in the lens cytoplasm, including protein aggregation. The present work continues a process of extending the initial work on liquid-liquid phase separation and related properties of aqueous $\gamma$-crystallin solutions to successively more realistic mixtures of eye lens crystallins. ${ }^{28,30-32}$

It is important to discuss the relationship of this work to investigations of eye lens proteins that are most commonly carried out at relatively dilute concentrations in comparison with those studied here. For example, the recognition of chaperone properties of the lens crystallins and numerous subsequent biophysical, chemical, and genetic studies have considerably enhanced our understanding of lens protein aggregation. ${ }^{33-35}$ In both equilibrium and kinetic contexts, dilute and semidilute solution investigations are critical for determining key structural aspects of proteins, for identifying specific interactions between proteins, and for quantifying their strength. More specifically, it is clearly critical to determine the free energies and kinetics of formation of prevalent complexes of lens proteins, including their dependence on protein sequence, chemical modifications, and solution conditions, and such investigation is most readily carried out without the added complexities of highly concentrated mixtures.

However, at the same time, it has long been very clear that the high crystallin concentrations in eye lens cells produce emergent mixture properties, including phase transitions, that are at present very difficult to predict solely from knowing dilute solution properties. Therefore, both dilute and concentrated solution investigations are essential and indeed complementary for understanding the molecular driving forces that underly lens protein aggregation, phase separation, and, ultimately, cataract.

In a previous paper, ${ }^{32}$ we showed that our experiments and numerical simulations strongly support the hypothesis that there is a weak attraction between $\gamma$-crystallins and $\alpha$-crystallins and that this attraction enhances the stability of mixtures of $\gamma$ and $\alpha$ with respect to phase separation at high concentrations, concentrations comparable to those in the eye lens. As a result, we obtained a coarse-grained model of this binary mixture. In this paper, we discuss further details of the model. In particular, we focus on the structural properties of the mixtures as obtained from the simulations. Finally, we use thermodynamic perturbation theory to describe the stability of the mixtures in the full parameter space.

The paper is organized as follows. In section II, the SANS experiments on a solution of crystallins are reported, and the colloidal behavior of the mixtures is discussed qualitatively. Section III is dedicated to the molecular dynamics simulations performed and to the derivation of a coarse-grained model of the proteins that is able to account for the experimental spectra. The influence of the $\alpha$-crystallin $-\gamma$-crystallin interaction on the stability of their concentrated mixtures, as deduced from the present coarse-grained model, is described, and possible origins of these interactions are discussed. Finally, in section IV, a statistical-thermodynamic perturbation theory for $\alpha-\gamma$ mixtures is devised, guided by the validated, coarse-grained model developed by comparison of molecular dynamics with experiment. Due to its analytic nature, such a perturbation theory considerably facilitates investigation and prediction of how crystallin protein properties affect the chemical potentials, light scattering, and phase separation crucial to understanding eye lens cytoplasm.

\section{Experiments}

Small-angle scattering experiments were performed on solutions of $\alpha$ - and $\gamma \mathrm{B}$-crystallins obtained by mixing different ratios $C_{\alpha}$ of a $230 \mathrm{mg} / \mathrm{m} l \alpha$-crystallin and a $260 \mathrm{mg} / \mathrm{mL} \gamma \mathrm{B}$-crystallin stock solution in $0.1 \mathrm{M}$ sodium phosphate buffer in $\mathrm{D}_{2} \mathrm{O}$ at $\mathrm{pH}$ 7.1 , with $20 \mathrm{mM}$ dithiothreitol to inhibit protein oxidation and oligomerization. We define $C_{\alpha}$ as the relative volume of the $\alpha$ solution according to

$$
C_{(\alpha)}=\frac{V_{(\alpha)}}{V_{(\alpha)}+V_{(\gamma \mathrm{B})}}
$$

with $V_{(i)}$ denoting the volume of the protein solution of type $i$. In the following, $\gamma \mathrm{B}$-crystallins will be simply denoted as $\gamma$.

The concentration of the $\gamma$-crystallin corresponds closely to their critical concentration $C_{\mathrm{c}}$, and all experiments were performed at a temperature of $25^{\circ} \mathrm{C}\left(10{ }^{\circ} \mathrm{C}\right.$ above the critical temperature $T_{\mathrm{c}}$ of the $\gamma$ 's in the present buffer).

The SANS spectra of the pure $\alpha$ - and pure $\gamma$ solutions together with the binary $C_{\alpha}=0.125,0.25$, and 0.5 mixtures are plotted in Figure 1 and cover the $q$ range of $0.002-3 \mathrm{~nm}^{-1}$. The pure $\gamma$ solution is already highly critical due to the proximity of the critical point of the gamma solution. $T$ is indeed just above the critical temperature $T_{\mathrm{c}}^{\gamma}$ of the $\gamma$ 's, and the density of the solution is near to the critical one. Adding $\alpha$-crystallins to the $\gamma$ solution first slightly increases the forward scattering $\left(C_{\alpha}\right.$ $=0.125)$. The intensity at low $q$ reaches a maximum for a mixing ratio of $C_{\alpha}=0.25$, while the peak due to the hard-core of the $\alpha$-crystallins appears at $q_{\alpha}^{*} \approx 2 \pi / d_{\alpha}$. Thus, a first estimate 


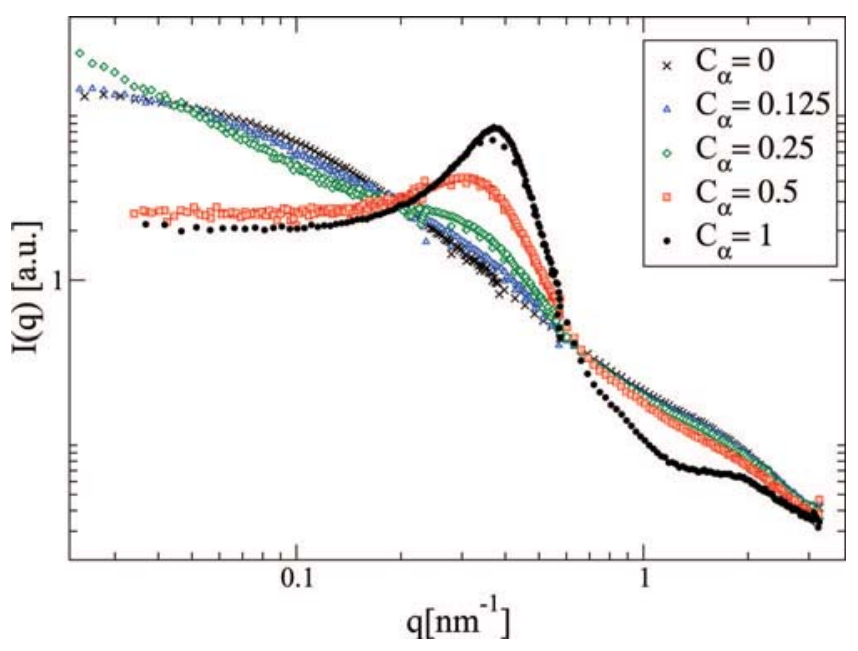

Figure 1. Small-angle neutron scattering intensity $I(q)$ versus wavevector magnitude $q$ for high-concentration bovine $\gamma \mathrm{B}$ - and $\alpha$-crystallin mixtures, showing evolution from pure $\gamma \mathrm{B}$ to $\alpha$. Mixing ratios $C_{\alpha}$ of a $230 \mathrm{mg} / \mathrm{mL} \alpha$-crystallin $\left(C_{\alpha}=1\right)$ and a $260 \mathrm{mg} / \mathrm{mL} \gamma$-crystallin stock solution are shown as follows: pure $\alpha$-crystallin, that is, $C_{\alpha}=1$ (dots), 0.5 (square), 0.25 (diamond), and 0.125 (triangle); pure $\gamma$-crystallins, that is, $C_{\alpha}=0$ (triangle left).

of the $\alpha$ 's diameter can be inferred from the experimental spectra, and the $d_{\alpha} \sim 160 \AA$ that we measure is consistent with previous studies. ${ }^{18}$

For $C_{\alpha}=0.5$, the large-scale fluctuations are already highly suppressed, and the pronounced structure factor peak of the $\alpha$-crystallins is found.

Qualitatively, as we have described, these measurements on high-concentration mixtures show a combination of the key features of hard-core repulsion and short-range attraction that are relevant in the pure solutions. However, since quantitative models of interactions between proteins within mixtures cannot be inferred directly from the SANS experiments, especially for the interaction between unlike components, the next section is dedicated to building a quantitative, testable model for the relevant lens protein interactions with the aid of computer simulations.

\section{Simulations and Model Validation}

A. Model. The proteins are modeled as particles that have a simple short-range interaction. Each protein is treated as an additive hard sphere with square-well (SW) interaction. Consequently, the interaction potential is defined as

$$
U_{i j}(x)= \begin{cases}\infty & \text { if } x<d_{i j} \\ -u_{i j} & \text { if } d_{i j}<x<d_{i j}+\lambda_{i j} \\ 0 & \text { if } x>d_{i j}+\lambda_{i j}\end{cases}
$$

with $d_{i j}=\left(d_{i}+d_{j}\right) / 2$ and $d_{i}$ as the diameter of the $i$ species. The parameters $u_{i j}$ and $\lambda_{i j}$, defining the energy scale and the range of the interactions, respectively, are the key quantities of our model and will be fixed using the experimental spectra.

The interaction potential defined by eq 2 clearly represents a strong idealization of the true interaction between the proteins. All of the internal degrees of freedom of the proteins and the directionality of their interaction are ignored.

In principle, an atomistic model that takes into account the internal structure of the two proteins could be studied by simulations. This approach, however, would be limited by the computational overhead to just a few proteins. Since we are interested in capturing the experimental phenomenology in a semiquantitative manner, which requires considering collective properties on length scales much larger than the single proteins, the atomistic simulation route is not feasible. An atomistic model is also not an option since, while the structure of the $\gamma$-crystallins is known, the same is not yet true for the structure of the $\alpha^{36,37}$

A second advantage of our model is that it comprises a minimal set of parameters necessary to account for the key phase equilibrium and scattering properties observed to date in aqueous solutions of pure $\alpha$, pure $\gamma$, and their binary mixtures. Moreover, each model parameter has a specific physical interpretation. The SW potential, in particular, has already been successfully used to model the phase behavior of $\gamma$-crystallins. ${ }^{23}$ The choice of SW potential is certainly not unique, and other shapes of the potential could also be considered. However, when the range of a spherically symmetric model attraction is smaller than the diameter of the particles, the phase diagram, liquid structure, and dynamics of the system are scarcely dependent on the exact shape of the potential, the second virial coefficient being the relevant parameter for both statics and dynamics. ${ }^{38,39}$ Finally, SW potentials permit fast simulations and simplify significantly the calculations within the perturbative approach that will be discussed in the last section.

B. Simulations. We performed standard molecular dynamics (MD) simulations in a cubic box with periodic boundary conditions for $N=32000$ particles (64000 for the pure $\gamma$ case). The algorithm follows the usual event-driven scheme for particles interacting via stepwise potentials, in which the trajectories of the different components are propagated from one interaction to the next. ${ }^{40}$ For each state point, up to six initial configurations were carefully equilibrated to the desired temperature by coupling the system to a thermostat. Once the system equilibrated, the production runs were performed by constant energy simulations. The scattering intensities were then computed from the configurations stored during these runs. To improve the statistics, the results of different independent runs were averaged, reducing significantly the error in the calculation of the scattering intensities, especially at low $q$ vectors. During the production runs, the temperature and pressure were monitored to check that no phase separation or crystallization was occurring. In some cases, equilibration was not possible, the system being intrinsically out of equilibrium (see the derivation of the model, section III.C). This was an indication that a spinodal line/surface had been crossed. In this case, the measured scattering intensities were taken while the system was still coupled to the thermostat.

1. Partial Structure Factors and Scattering Intensities. Scattering techniques are one of the most important approaches to study the properties of soft matter. Depending on the length scales to be resolved and the contrast between suspended particles and solvent, the scattering of neutrons, X-rays, or light is used. Experiments performed in the infinite dilution limit, that is, at very low concentration, give access to quantities directly related to the size and shape of the suspended particles, while at higher concentration, we can gain information about particle arrangements and interactions. In the latter case, the relevant quantity is the static structure factor $S(q)$ that is defined as the equal time density-density correlation and reads for homogeneous systems

$$
S(q)=\frac{1}{N}\left\langle\sum_{i, j} \mathrm{e}^{\mathrm{i} \mathbf{q} \cdot\left(\mathbf{r}_{i}(t)-\mathbf{r}_{j}(t)\right)}\right\rangle
$$

where the sum runs over the $N$ particles of the system and the

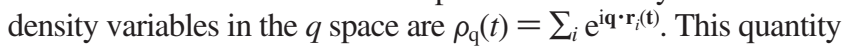


is directly related by Fourier transform to the radial distribution function $g(r)$. By measuring first the form factor $I_{0}(q)$, the scattering intensity of a dilute sample of $N_{0}$ particles for which all correlations between the particles have been minimized, $S(q)$, can be easily evaluated from the scattering intensity $I(q)$ by computing the ratio ${ }^{41}$

$$
\frac{I(q)}{I_{0}(q)}=\frac{N}{N_{0}} S(q)
$$

For a mixture of different components, the scattering experiments provide the total intensity scattered by the mixture, without distinguishing the contributions of the different components. In other words, the partial structure factors and the related partial radial distribution functions cannot be measured straightforwardly.

These quantities, however, can be directly accessed by numerical simulations and integral equations calculations, ${ }^{42}$ and they are related to the total scattered intensity measured in the experiments. The scattered intensity versus the scattering vector $q$ for a mixture of $m$ components can be expressed as ${ }^{41}$

$$
I(q)=N \sum_{\mu, v=1}^{m} f_{\mu}(q) f_{\nu}(q) S_{\mu \nu}(q)
$$

in which $f_{\mu}(q)$ denotes the form factor of particles of species $\mu$ and $S_{\mu, \nu}(q)$ is the partial static structure factor

$$
S_{\mu \nu}(q)=\frac{1}{N}\left\langle\sum_{i=1}^{N_{\mu}} \sum_{j=i}^{N_{\mu}} \mathrm{e}^{\mathrm{i} \mathbf{q} \cdot\left(\mathbf{r}_{i \mu}(t)-\mathbf{r}_{j \nu}(t)\right)}\right\rangle
$$

For a the binary $\alpha-\gamma$ mixture, the scattering intensity reads

$$
\frac{I(q)}{N}=f_{\alpha}^{2}(q) S_{\alpha \alpha}(q)+f_{\gamma}^{2}(q) S_{\gamma \gamma}(q)+2 f_{\alpha}(q) f_{\gamma}(q) S_{\alpha \gamma}(q)
$$

In this work, the total scattering intensity has been calculated by eq 7 with the partial static structure factors $S_{\mu \nu}(q)$ obtained from the simulations and the form factors $f_{\mu}(q)$ of the two species measured experimentally.

The static structure factors $S_{\mu \nu}$ were calculated by averaging over several independent configurations and runs. To improve the statistics, averages over 300 different wave vectors $\mathbf{q}$ of the same modulus $q$ were also performed. The experimental form factors were fitted using indirect fourier transformation ${ }^{43}$ (IFT) to get rid of the experimental noise at high $q$ and Guinier fit to have access to low q vectors. ${ }^{44}$ The results are shown in Figure 2. In order to account for the experimental smearing, we derived a general resolution function resulting from the combined effect of wavelength spread, finite collimation, and detector resolution ${ }^{45}$ (see Appendix A). The simulated scattering intensities were convoluted using this resolution function before comparing them with the SANS data.

C. Derivation of the Model. In this section, we shall describe the steps that we have followed to derive the parameters in the model introduced by eq 2 . We will start by studying the pure solution of the two proteins. In this way, we will derive the parameters of the pure components, that is, the attractive square-well depths $\left(u_{\alpha \alpha}\right.$ and $\left.u_{\gamma \gamma}\right)$ and the ranges $\left(\lambda_{\alpha \alpha}\right.$ and $\left.\lambda_{\gamma \gamma}\right)$. The second more challenging step will be toward modeling the

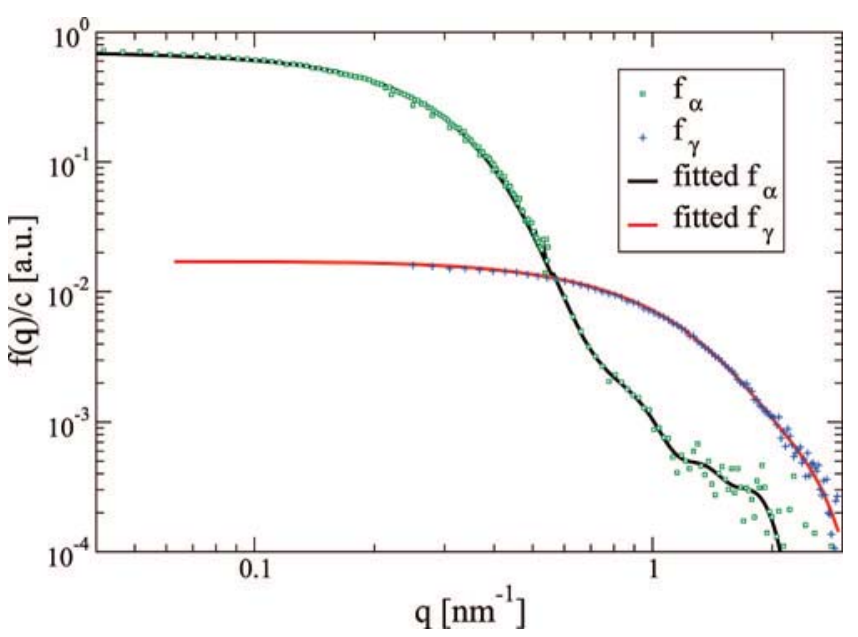

Figure 2. Concentration-normalized scattered intensities of diluted $\alpha$-crystallin (squares) and $\gamma$-crystallin (plus) solutions (4 and $8 \mathrm{mg} /$ $\mathrm{mL}$ ) that were used as the experimental form factors. Also shown are the IFT fits (solid lines).

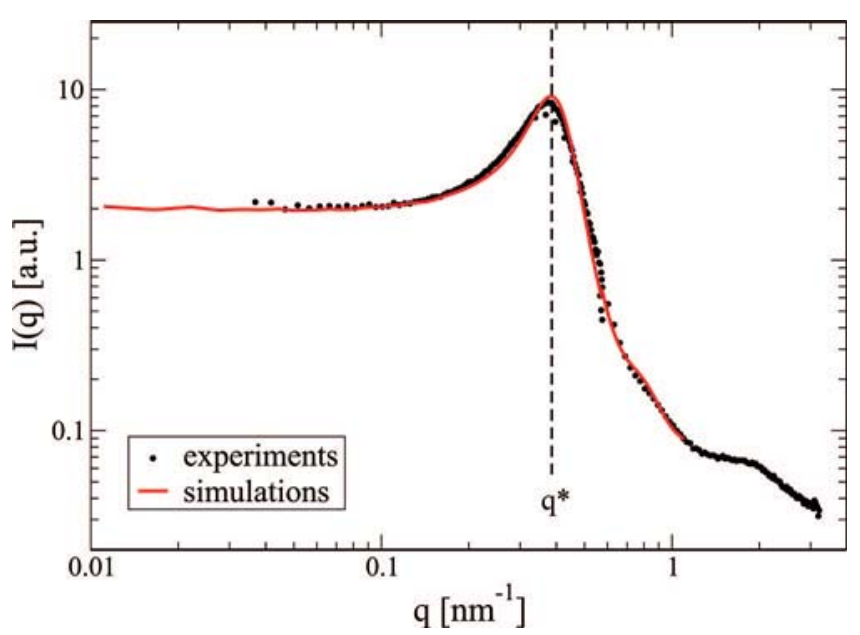

Figure 3. Neutron scattering intensities for the concentrated, pure $\alpha$-crystallin solutions in the present buffer are well-modeled with use of a hard-sphere potential in molecular dynamics simulations. SANS intensities (dots) and MD results (line) for hard spheres at a packing fraction of $\phi_{\alpha}=0.39$ are shown. The location of the peak $q^{*} \sim 2 \pi / d_{\alpha}$ $=0.385 \mathrm{~nm}^{-1}$ is also drawn.

binary mixtures. In this case, no prior studies were available; the interactions between unlike components were unknown and could not be inferred from the single-component behavior.

1. Pure Component Mixture. The experimental scattering intensity $I(q)$ as a function of the scattering vector $q$ for the $\alpha$-pure case is shown in Figure 3. The main features are a low forward scattering $I(q \longrightarrow 0)$ and a peak at around $q \sim 0.038 \AA^{-1}$. In agreement with previous studies, ${ }^{18,46}$ we assume the interactions between $\alpha$-crystallins as purely repulsive. In our model, this means that we model them as hard spheres. If the $\alpha$-crystallin molecular weight is taken to be $8 \times 10^{5} \mathrm{~g} / \mathrm{mole}$ and it is taken to have a radius of $81.5 \AA$, consistent with our observations, the volume occupied per unit weight of $\alpha$-crystallins is found to be $1.7 \mathrm{~mL} / \mathrm{g}$. This value is in agreement with the partial occupied volume of bovine $\alpha$-crystallin determined in previous studies. ${ }^{53} \mathrm{We}$ used it to determine the volume fraction $\phi_{\alpha}=0.39$ corresponding to the pure $230 \mathrm{mg} / \mathrm{mL}$ stock solution. By comparison of the SANS results and the MD simulations, an effective diameter of $d_{\alpha}=163 \AA$, compatible with the values found in literature ${ }^{18}$ is obtained. 
A satisfactory agreement between the experimental and simulated intensities $I(q)$ is obtained as shown in Figure 3. In terms of structure, the peak can be interpreted as the enhanced correlations at the contact distance between the particles. The location of the peak $q^{*}$ varies with the diameter as $q^{*} \sim 2 \pi / d_{\alpha}$. Even though the $\alpha$-crystallins are globular proteins composed of many subunits and the solution is expected to be slightly polydisperse ( $15 \%),{ }^{46}$ the assumption of monodispersity used in this study is sufficient to recover the form of the experimental $\alpha-\alpha$ correlation peak at this intermediate density. The polydispersity is expected to improve the agreement in the shape of the contact peak, and it could be considered in future developments of the model.

Now, we turn our attention to the $\gamma$ solution. Aqueous solutions of $\gamma$-crystallins have attracted considerable interest in the past years as model systems for the study of phase transition and critical phenomena. ${ }^{19}$ These solutions become turbid when cooled below a certain critical temperature which depends on the experimental buffer. Strong density fluctuations that occur near a critical point are responsible for a fluid-fluid phase separation of the solution. More specifically, a coexistence curve divides the density-temperature plane into a hightemperature region, where a single homogeneous phase exists, and a low-temperature one where two liquid phases of different density are in equilibrium. Due to the different refractive index between the phases at high and low concentrations, the solution appears opaque. Previous experiments led to the determination of the coexistence curve and critical point $\left(\phi_{\mathrm{c}}, T_{\mathrm{c}}\right)$ of the $\gamma$ solutions, demonstrating the presence of a second-order liquid-liquid phase transition. ${ }^{19}$ While the exact location of the coexistence curve depends on the solvent and on the particular $\gamma$-crystallin being investigated, the solution properties have been found to scale with the reduced density and the reduced temperature $\left(T-T_{\mathrm{c}}\right) / T_{\mathrm{c}} \cdot{ }^{19,20,26,27}$

The first parameter that we shall set is the range for the $\gamma-\gamma$ interaction. This is fixed to reproduce the critical density obtained by the experiments. Square-well systems have been intensively studied by means of Monte Carlo (MC) simulations, and their coexistence curve has been computed for a large spectrum of attraction ranges. ${ }^{47}$ In our case, a range $\lambda_{\gamma \gamma}=0.25 d_{\gamma}$ produces the correct critical density. Indeed, MC simulations with this value of the short-range attraction gave good agreement with the experimental coexistence curve. ${ }^{23}$

In order to enhance the fluctuations, the experiments were performed at $25^{\circ} \mathrm{C}$, close to $T_{\mathrm{c}}=15{ }^{\circ} \mathrm{C}$, the critical temperature of $\gamma$ in the present buffer. The concentration of the $\gamma$-pure solution used here is close to the critical one, that is, $c_{\mathrm{c}}=260$ $\mathrm{mg} / \mathrm{mL}$, and corresponds to a packing fraction of $\phi_{\gamma}=\phi_{\gamma}^{\mathrm{c}}=$ 0.184 , given a specific volume for the $\gamma$-crystallins of $v=0.71$ $\mathrm{cm}^{3} / \mathrm{g} \cdot{ }^{30}$

We performed the numerical simulations at this packing fraction using $\lambda_{\gamma \gamma}=0.25 d_{\gamma}$ for the square-well potential, with $d_{\gamma}=36 \AA$, the effective sphere diameter that reproduces the volume per molecule of the $\gamma$. Setting the energy parameter to $u_{\gamma \gamma}=1$, in units of $k_{\mathrm{B}}=1$, leaves the temperature $T$ as the only free parameter. For $\bar{T}=0.7875$, good agreement between the experimental and numerical $I(q)$ is found; see Figure 4. The intensities show a strong forward scattering at large length scales (low $\mathrm{q}$ vectors), which is a direct consequence of the strong density fluctuations in the proximity of the critical point. Indeed, the structure factor $S(q)$ (proportional to $I(q)$ at low $q$ ) is defined as the density-density correlation function in Fourier space, and the strong scattering is the result of long-wavelength density fluctuations in the sample. These strong fluctuations are

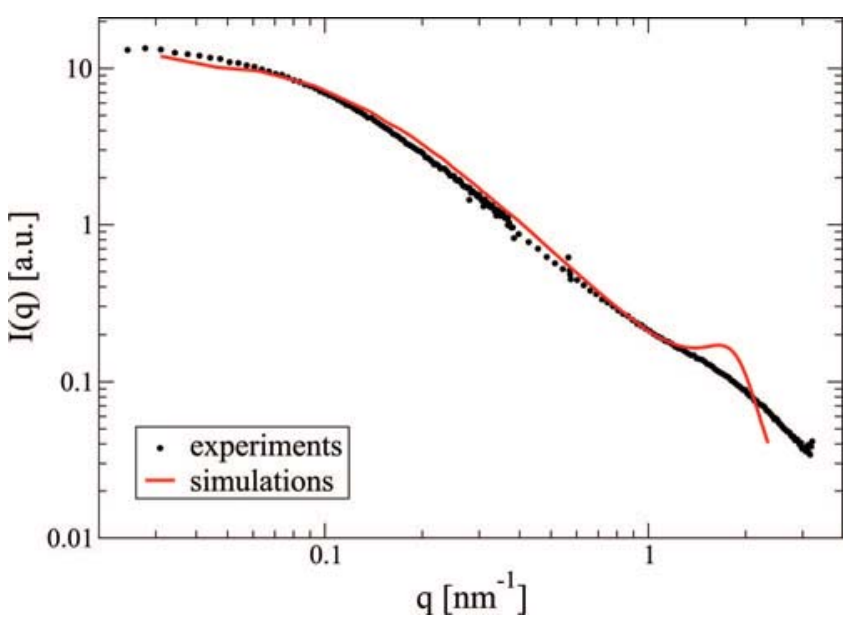

Figure 4. Neutron scattering intensities for the concentrated, pure $\gamma$-crystallin solutions in the present buffer can be approximated for wavevector magnitudes below $1 \mathrm{~nm}^{-1}$, with use of a spherical squarewell potential in molecular dynamics simulations. SANS measurements (dots) and results from molecular dynamics simulations (line) for square-well particles $\left(\lambda_{\gamma}=0.25 d_{\gamma}, u_{\gamma \gamma}=1, \bar{T}=0.7875\right)$ at a packing fraction of $\phi_{\gamma}=0.18$ are shown.

particularly important for the physiological function of the eye lens proteins, which is to ensure the eye lens transparency. Benedek in his seminal paper Theory of Transparency of the Eye demonstrated that the turbidity of the cataractous eye lens is related to microscopic spatial fluctuations in its index of refraction..$^{16}$ In particular, the scattering of light is produced primarily by those fluctuations whose Fourier components have a wavelength equal or larger than half of the wavelength of light in the medium. The low $q$ regime relevant for eye lens transparency is thus defined as $q \lesssim 0.047 \mathrm{~nm}^{-1}$, and particular care has been taken to reproduce the intensity in this region.

Not surprisingly, the agreement at high $q$ values is less satisfactory due essentially to the appearance of a nearestneighbor peak at $q^{*} \sim 2 \pi / d_{\gamma}$ in the simulation data. Contrary to the $\alpha$-crystallins, $\gamma$ has been crystallized, and its structure has been resolved. ${ }^{36,37,48,49}$ It consists of two distinct domains that form a slightly elongated ellipsoid. The discrepancy between simulation and experiment at high $q$ might arise from the simplifying assumption of a spherical shape. In particular, the contact peak for ellipsoidal particles would be less pronounced due to the broadened distribution of the contact lengths.

Another essential feature of globular proteins that is not considered in the present model and which may well affect the high $q$ scattering is the strong directionality of the interactions. Proteins are characterized by patchy surfaces that are indeed typically essential for their biological roles. This has motivated the introduction of nonisotropic models of protein phase behavior. Patchy, or aeolotopic, models that consider protein directional interactions have been able to address phenomena such as protein aggregation, crystallization, and self-assembly. ${ }^{50-52}$

In this work, however, we shall show that even if an isotropic model of attraction represents a strong simplification, it is certainly adequate for our purpose since it reproduces the key features on the longer length scales of interest for transparency. The introduction of anisotropy, both in shape and interactions, might be an interesting refinement for further studies.

2. Binary $\alpha-\gamma$ Mixture. Inferring the microscopic interactions between proteins from scattering experiments becomes more complicated when dealing with systems composed of more than one component. The factorization of the scattering intensity $I(q)$ into a one-particle property and a genuine statistical 


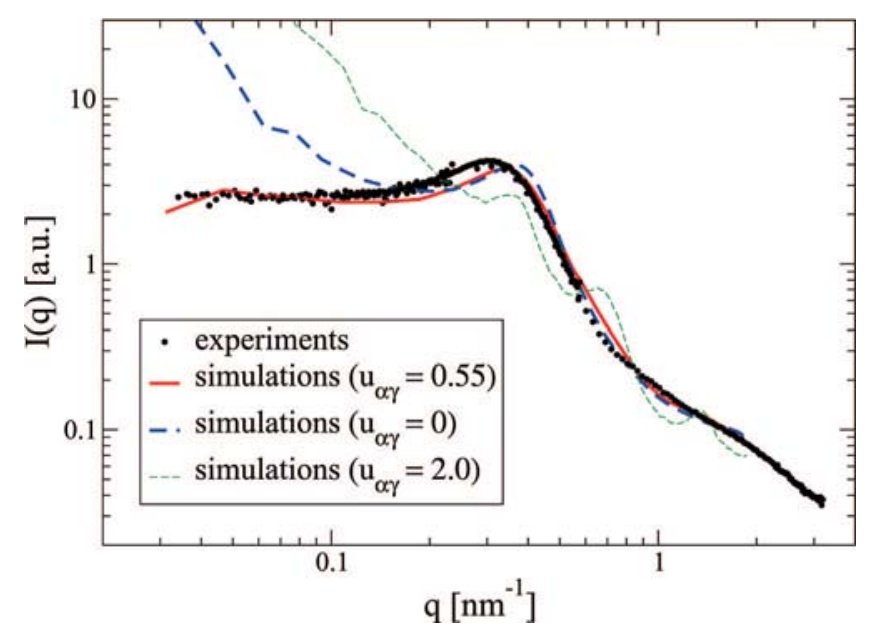

Figure 5. Attraction between $\alpha$ - and $\gamma$-crystallins is essential for modeling neutron scattering intensities of their high-concentration mixtures. ${ }^{32}$ SANS intensities for the $C_{\alpha}=0.5$ mixture (dots) and results from molecular dynamics simulations at $\bar{T}=0.7875$ are shown for three different values of the interspecies interaction $\left(u_{\alpha \gamma}=0, u_{\alpha \gamma}=\right.$ 0.55 , and $u_{\alpha \gamma}=2$ denoted by the dashed, the full, and the dotted line, respectively).

mechanical quantity (see eq 4), like the structure factor $S(q)$, is no longer possible. As expressed by eq 7, the measured intensity is a result of a combination of the form factors and partial structure factors $S_{\mu, v}(q)$ of each component. The latter cannot be isolated by simply knowing $I(q)$. The MD simulations now become essential in the present context for understanding the microscopic interactions between the components that lead to the observed experimental scattering intensities of the mixtures.

Among the three different mixing ratios $\left(C_{\alpha}=0.5,0.25\right.$, and $0.125), C_{\alpha}=0.5$ closely resembles the natural $\alpha-\gamma$-crystallin concentration found in the eye lens nucleus. ${ }^{53}$ Therefore, the model parameters for the mixtures are derived on this sample and then tested and confirmed also for the remaining mixing ratios. The temperature $\bar{T}$ at which the simulations on the mixtures are performed is fixed by the pure $\gamma$ case. The remaining free parameter is the interaction between unlike proteins $u_{\alpha \gamma}$ that will turn out to be essential for understanding the stability of the binary mixture.

Since no previous studies of the present type for the interaction between the two crystallin proteins are available, we start with the assumption of a purely repulsive additive hardsphere interaction between the two components, that is, $u_{\alpha \gamma}=$ 0 . The striking disagreement between the forward scattering of the numerical results and the experimental data for $C_{\alpha}=0.5$ is evident in Figure 5. The experimental $I(q)$ resembles closely the pure $\alpha$ case with a peak at intermediate $q$ range and a highly suppressed forward scattering (Figure 1). Considering hardsphere repulsion among the two species only results in an enormous increase of the scattering intensity at low $q$, which can be understood as an enhancement of the unstable region of the mixture. Indeed, we were not able to properly equilibrate the mixture during molecular dynamics simulations. The system began to phase separate, forming large domains of $\alpha$-crystallinrich and $\gamma$-crystallin-rich regions, as clearly indicated from the snapshots presented later. In the real system, these effects usually start to emerge in $\alpha-\gamma$ mixtures at temperatures above the $\gamma$ critical temperature that are somewhat lower than those investigated here. ${ }^{28}$ As a consequence of the high forward scattering, the transparency of the mixture would be lost, in contrast with the visual appearance of the sample and the SANS data, which

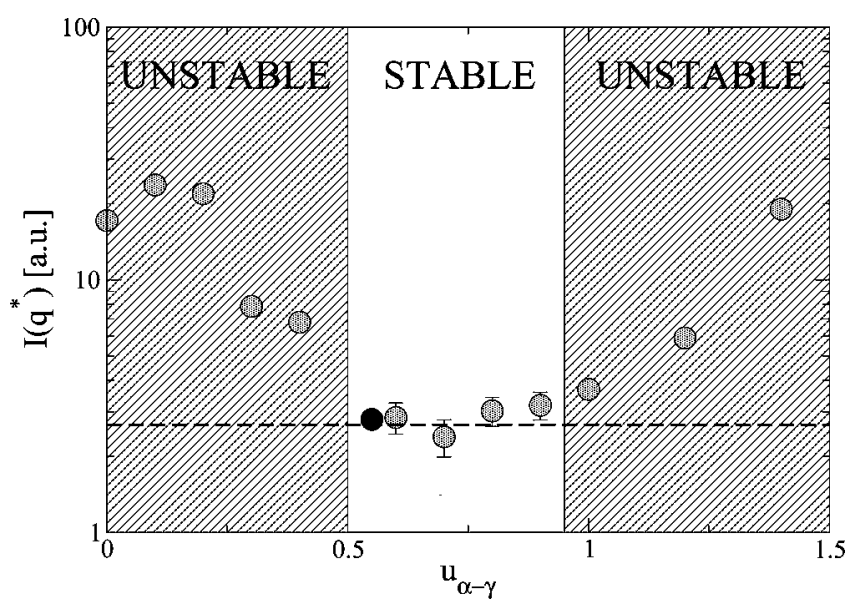

Figure 6. A narrow range of the attraction strength between $\alpha$ - and $\gamma$-crystallins is compatible with avoiding high-concentration phase separation instability. Dots show calculated scattering intensities for $q^{*}=0.0467 \mathrm{~nm}^{-1}$ from analysis of molecular dynamics simulations versus the $\alpha-\gamma$ attraction square-well depth $u_{\alpha \gamma}$ for the $C_{\alpha}=0.5$ mixture (see text). The attraction range in which the mixture remains stable against phase separation and can be equilibrated is also drawn. The dark point indicates the value of $u_{\alpha \gamma}$ that we used to model the $\alpha-\gamma$ mixture, and the dashed line represents the experimental $I\left(q^{*}\right)$.

TABLE 1: Parameters of the Square-Well Potential Used for Modeling the $\alpha-\gamma$ Mixtures $^{a}$

$\begin{array}{llllll}d_{\gamma} & 1.0 & \lambda_{\gamma} & 0.25 d_{\gamma} & u_{\gamma \gamma} & 1.0 \\ d_{\alpha} & 4.53 & \lambda_{\alpha} & 0.0 & u_{\alpha \alpha} & 0.0 \\ d_{\alpha \gamma} & 2.765 & \lambda_{\alpha \gamma} & 0.25 d_{\gamma} & u_{\alpha \gamma} & 0.55\end{array}$

${ }^{a}$ The diameters $d$ and ranges of the interaction $\lambda$ are given in units of $d_{\gamma}=36 \AA$, and the depth of the potential $u$ is in units of $k_{\mathrm{B}}$ $=1$. The temperature of the simulations was set to $T^{*}=0.7875$.

indicate that under the present conditions, fluctuations at low $q$ are highly suppressed.

It is clear that some element that stabilizes the mixtures has not been considered in this first model. Consequently, we speculate that a mutual attraction between unlike proteins might be present to circumvent these long-wavelength fluctuations and to stabilize the mixtures. We assume this attraction to have the same range as the one used for the attraction between $\gamma$-crystallins $\left(\lambda_{\alpha \gamma}=\lambda_{\gamma \gamma}=0.25 d_{\gamma}\right)$.

To pin down an effective well depth for this mutual attraction, we performed several simulations varying $u_{\alpha \gamma}$ from the hardsphere limit up to 2 . The stability of the binary mixtures appears to depend on $u_{\alpha \gamma}$ in an extremely sensitive and nonmonotonic way. To illustrate this point, we plot in Figure 6 the scattering intensity for a q vector in the region relevant for transparency $\left(q^{*}=0.0467 \mathrm{~nm}^{-1}\right)$ as a function of the $\alpha-\gamma$ attraction for $C_{\alpha}$ $=0.5$. The system can be equilibrated for $0.5<u_{\alpha \gamma}<1$, and a low forward scattering, compatible with the experiments, is obtained for these values of $u_{\alpha \gamma}$. Outside of this interval, the simulations show a clear sign of phase separation, which indicates the crossing of an unstable region. The strong fluctuations at low $q$ that take place when an instability boundary is reached are responsible for the enhancement of $I\left(q^{*}\right)$. The origin of these fluctuations and the nature of the phase separation will be investigated in the next sections. We found that an interspecies attraction of half of $u_{\gamma \gamma}\left(u_{\alpha \gamma}=0.55 u_{\gamma \gamma}\right)$ is sufficient to account for the SANS measurements and accurately reproduces the scattering intensities at low $q$. The parameters of our model of the $\alpha-\gamma$ mixtures are summarized in Table 1. For this choice, a simulated scattering intensity in good agreement with the experiments is obtained for $C_{\alpha}=0.5$; see Figure 5 . 

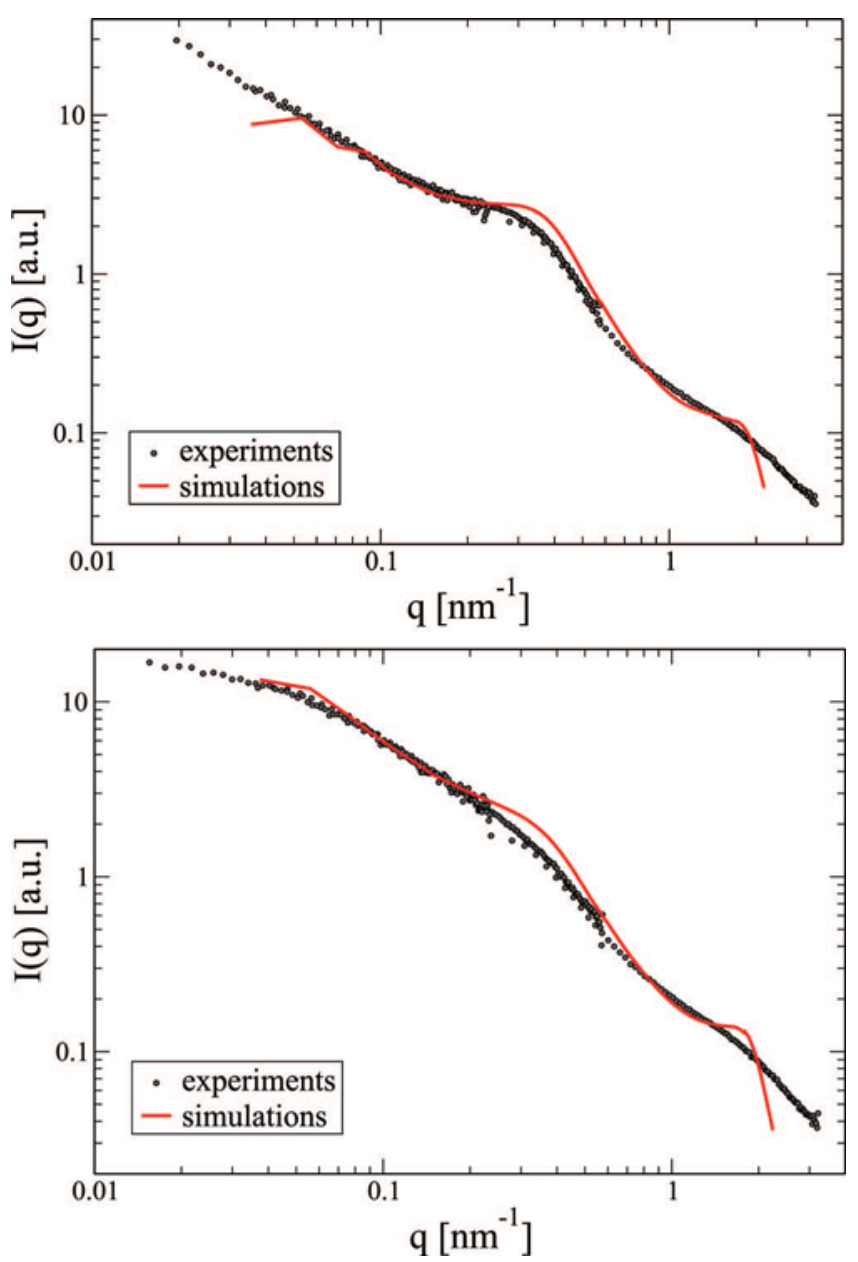

Figure 7. The neutron scattering intensities for the $C_{\alpha}=0.25$ (top) and 0.125 (bottom) mixtures are compatible with the $\alpha-\gamma$ square-well depth determined by comparison of MD simulations with the $C_{\alpha}=$ 0.5 SANS measurements. SANS measurements are represented by dots and results from molecular dynamics simulations by full lines. The parameters of the simulated model are presented in Table 1 .

Use of this attraction leads to a good description of the experimental $I(q)$ also for the remaining mixing ratios $\left(C_{\alpha}=\right.$ 0.25 and 0.125 ), as reported in Figure 7. The agreement for moderate $q$ vectors is less satisfactory. As for the pure mixtures case, this could be due to both polydispersity and anisotropy. In the $q$ region relevant for transparency, however, our simplified model gives a good account of the experimental results.

We have shown that this mutual attraction improves drastically the agreement with the experimental results. Its origin, however, remains unclear. In general, the exact origin of shortrange attraction in globular protein systems including $\gamma$-crystallins and lysozyme ${ }^{54}$ is still a challenging and open question. Three-dimensional protein structures often display large exposed hydrophobic regions, which are likely to play a prominent role in the interparticle interactions. ${ }^{4,55}$ Beside hydrophobicity, which is still far from being understood and whose contribution is difficult to evaluate, electrostatics might be important. ${ }^{2}$ At $\mathrm{pH}$ $=7$, where the experiments have been performed, $\alpha$ - and $\gamma$-crystallins are oppositely charged. More specifically, $\alpha$-crystallins are expected to carry $Z_{\alpha}=-180$ electronic charges per molecule at the experimental $\mathrm{pH},{ }^{56}$ while for $\gamma$-crystallins, $Z_{\gamma}$ is close to $+2 e .^{57}$ This suggests that electrostatic interactions could be considered as one possible origin of the mutual interaction that we have introduced in the model to reproduce the experimental data.
To investigate this possibility further, if we treat the proteins as charged colloids, we can use a pairwise screened Coulomb potential as an interaction model, where the range of the electrostatic interactions is characterized by the Debye screening length $\kappa^{-1}{ }^{58,59}$ This approach has often been used in the study of protein solutions. ${ }^{59-61}$ In order to estimate the electrostatic contribution to the expected $\alpha-\gamma$ attraction, we evaluated this pairwise screened Coulomb potential with the experimental screening length $\kappa^{-1} \sim 6 \AA$ and compared it with the squarewell potential that we used for the simulations. We found that the screened Coulomb potential, evaluated in this fashion, could not account for the magnitude of the interaction derived from the simulations; in order to recover the same second virial coefficient, a $Z_{\alpha}$ of $-450 e$ would have been needed.

However, it should be noted that the approach just described is simply a start toward investigating the electrostatic contribution to $\alpha-\gamma$ interactions, as it is based on the hypothesis of a uniform charge distribution on the surface of a sphere and is also based on the use of dilute solution approximations to the nature of electrolyte solutions long recognized to give inaccurate thermodynamic predictions at higher electrolyte concentrations unless they are modified considerably. ${ }^{58,62-64}$ For proteins, charged groups are far from being uniformly distributed on the surface but rather are grouped in patches that form a complex mosaic. Neighboring proteins will rotate due to interactions between these patches and can also exchange protons with the solvent, and by both mechanisms, they spend more time in configurations that have lower potential energy. How readily these adjustments occur will depend on the nature of the patchiness, on the Debye length, on the temperature, and on the ease of charge regulation, that is, the ease with which local proton occupancy and thus protein surface charge can vary at a given $\mathrm{pH}$. Thus, the rotationally averaged electrostatic screened potential would tend to underestimate the attractions. In order to investigate the origin of the attraction and how it might be influenced by electrostatics, one should therefore consider anisotropic interprotein potentials, ${ }^{50,51}$ as well as charge regulation. ${ }^{61,65}$

The ability of the $\gamma$-crystallins to fit into spaces between $\alpha$ subunits, which are about the same size as the $\gamma$ 's, could also lower the potential. In this context, taking into account the multisubunit character of the $\alpha$ or introducing some negative additivity $\left(2 d_{\alpha \gamma}<d_{\alpha}+d_{\gamma}\right)$ could also be interesting refinements of the model. Clearly, these are all speculations, and the quantitative molecular origins of the mutual attraction remain unknown and are worthy of further experimental and theoretical investigation.

Before moving to the description of the structural properties, we want to stress that the intrinsic anisotropic nature of the interactions does not significantly affect our results at low $q$, where the fluctuations that disrupt eye lens transparency occur. Indeed, in this region, we can obtain a satisfactory agreement with the experiments by the simple isotropic potential defined by eq 2 . For $q$ vectors around the $\alpha$ peak, angular-dependent interactions might be considered to improve the results. Moreover, the intrinsic polydispersity of $\alpha$-crystallins, which has to play a role in the properties of the pure $\alpha$ solution at higher packing, might also sensibly broaden the peak according to intuitive arguments similar to those given above in the case of the possible influence of the ellipsoidal shape of the $\gamma$-crystallins.

3. Structural Properties. One of the benefits of computer simulations for the study of binary mixtures is that the partial structure factors and partial radial distribution functions can be 
predicted. The partial structure factors quantify Fouriertransformed spatial arrangements of the individual protein components in the solution, with respect to each pair of species. Partial structure factor predictions both augment small-angle neutron scattering data and help the design of experiments that use selective species labeling or contrast variation to enable measurement of the partial structure factors.

Since our model has been validated by comparison with the experiments, the structural properties obtained from the simulations can now give important predictions about the crystallinspecies-specific spatial organization of the real system. In Figure 8 , we reproduce the three partial structure factors obtained for the choice of parameters used to model the $\alpha-\gamma$ mixture (Table 1). From the structure factors, it is evident that an increase of the $\gamma$-crystallin concentration fluctuations at large scale, indicated by the enhancement of $S_{\gamma \gamma}(q)$ at low $q$, is responsible for the mixture high criticality not only in the pure $\gamma$ case but also for the binary mixtures. Compared to the monodisperse $\gamma$ solution, the presence of the larger $\alpha$ 's enhances the criticality of the $\gamma$-crystallin fluctuations. As observed for the experimental $I(q)$, the dependence of the critical behavior on the relative composition of $\alpha$ - and $\gamma$-crystallins is not monotonic. Indeed, for $C_{\alpha}=0.25, S_{\gamma \gamma}(q)$ presents the strongest enhancement of large length scale fluctuations. This nonmonotonic dependence of criticality on $\gamma-\alpha$ composition is consistent with the nonmonotonic dependence of cloud temperature on $\alpha-\gamma$ composition found previously. ${ }^{28}$

$S_{\alpha \alpha}(q)$ also shows enhancement of large length scale fluctuations when increasing the amount of $\gamma$-crystallins in the solution, but in a less dramatic way. We also calculated the partial structure factors for other values of the mutual attraction. Outside of the narrow interval of attraction strength shown in Figure 6, all of the structure factors present a strong increase at low $q$ that is ultimately responsible for the strong forward scattering in the $I(q)$ spectra. This is illustrated in Figure 5 for the case of weak $\left(u_{\alpha \gamma}=0\right)$ and strong attraction $\left(u_{\alpha \gamma}=2\right)$.

If we could experimentally modify the $\alpha-\gamma$ attraction without concomitantly changing other key interactions, the transparency of the sample in these high and low regimes would be lost. We anticipate that the nature of long-wavelength fluctuations is different in the two extreme cases of primarily compositional and primarily protein density phase separation, as will be apparent later by visual inspection of the simulation box.

The structure in real space can give better insight into the local composition of the solutions, in particular, for the spatial distribution of each species with respect to the other. For this reason, the partial radial distribution functions (RDF) for the different $C_{\alpha}$ are presented in Figure 9. For the RDF of $\alpha$-crystallins, the pure case exhibits the usual oscillations of a dense hard-sphere system; the spatial arrangement of the components alternates between regions of enhanced and reduced probability of finding a particle at a certain distance $r$ from another, compared to that for a uniform distribution.

The range of the spatial correlation between $\alpha$ 's increases when $\gamma$ 's are added to the solution, as shown by the longer decay of the initial peak in $g_{\alpha \alpha}(r)$. This more collective behavior is a clear sign that the mixtures are becoming closer to criticality. For the case $C_{\alpha}=0.5$, however, the scattering intensity does not show such a long decay, indicating strong fluctuations yet. This is due to the glue effect of the small attractive proteins that form complexes with the large ones, as indicated by the cartoon in Figure 9, and, therefore, prevent demixing. This effect appears more clearly when increasing $u_{\alpha \gamma}$ up to the limit of the stability region (see inset of Figure 9); the $\alpha-\alpha$ contact peak
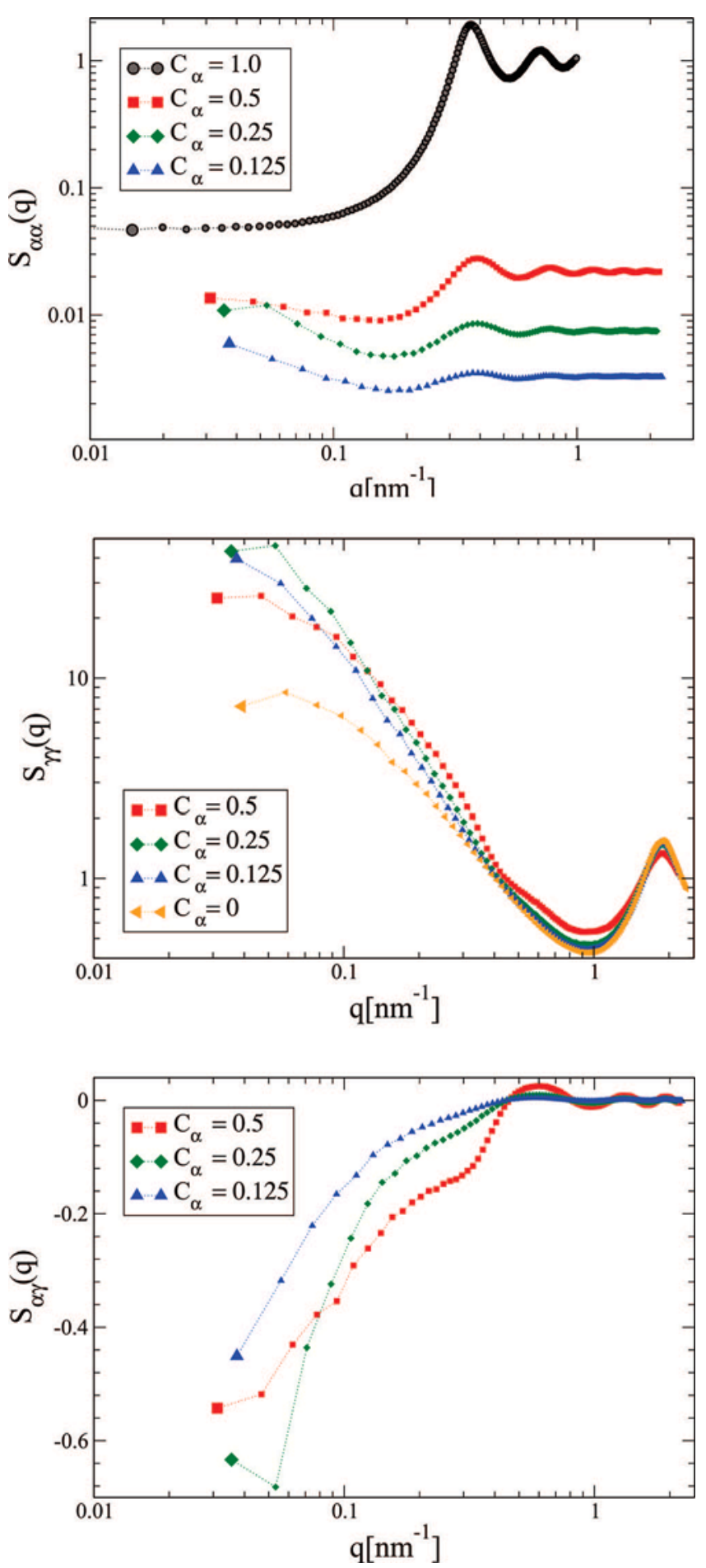

Figure 8. Predicted partial structure factors obtained from the MD simulations of the various $C_{\alpha}$ mixtures; (top) $S_{\alpha \alpha}$; (center) $S_{\gamma \gamma}$; (bottom) $S_{\alpha \gamma}$

is suppressed, while there is a strong enhancement of $\alpha-\gamma-\alpha$ configurations, as depicted in the cartoon of Figure 9.

A similar effect has been observed recently ${ }^{66}$ in asymmetric binary Yukawa fluids, where a weak attraction between small and big components leads to the formation of a shell of small particles around the large particles, which weakens the effective attraction between the large components and, thus, decreases the critical temperature of the mixture.

For the $\gamma$-crystallin RDFs, the attractive wells give rise to a pronounced enhancement of the probability of finding proteins close to each other; the proteins are likely to stay closer than 

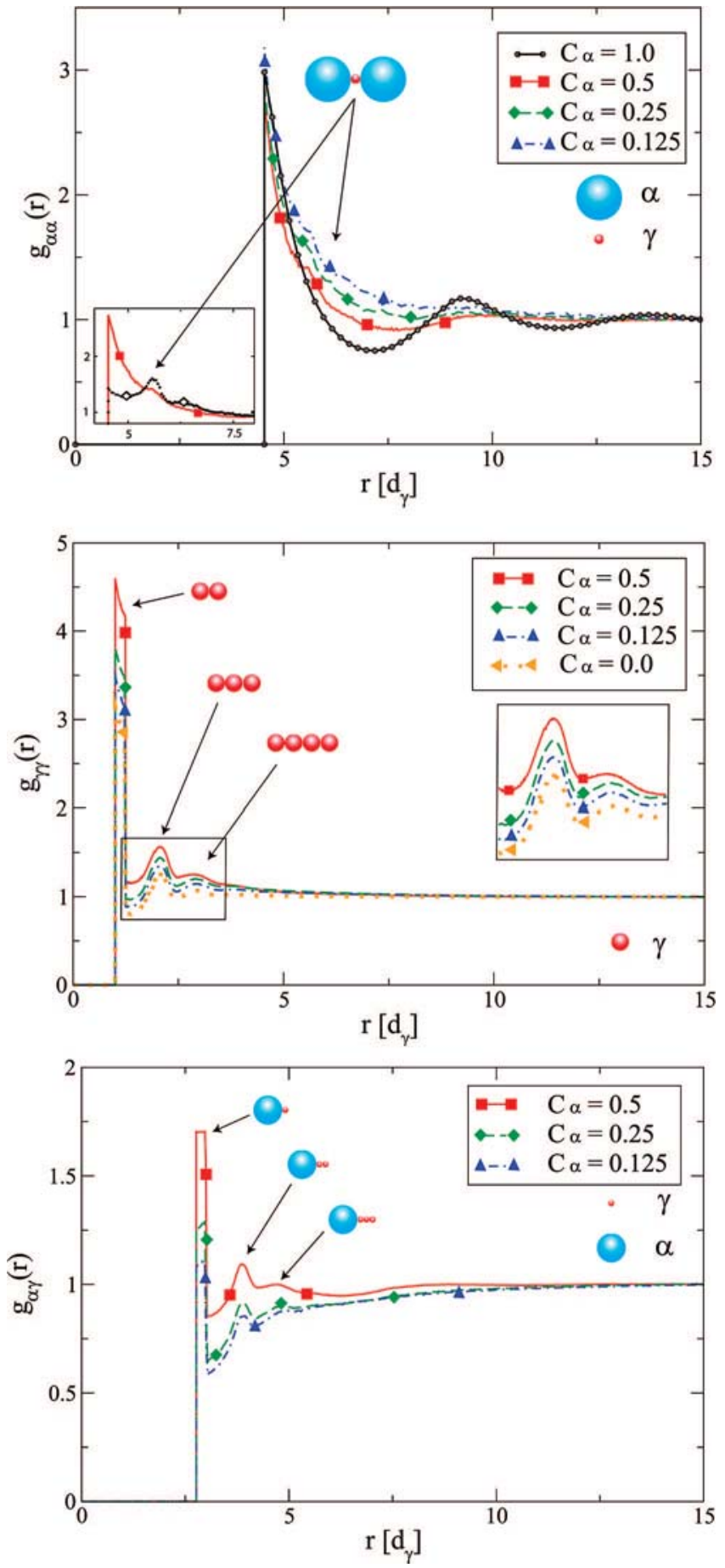

Figure 9. Partial radial distribution functions from MD simulations of concentrated $\alpha-\gamma$-crystallin mixtures, showing neighboring protein distributions by type as a function of mixing ratio, $C_{\alpha}$; (top) $g_{\alpha \alpha}(r)$; (center) $g_{\gamma \gamma}(r)$; (bottom) $g_{\alpha \gamma}(r)$. Key structural arrangements of the proteins that contribute to different peaks are also depicted (see text). The $g_{\alpha \alpha}(r)$ of the $C_{\alpha}=0.5$ mixture for $u_{\alpha \gamma}=0.55$ (line) and 0.9 (dark diamonds) are compared in the inset of the top panel.

the outer range of their attractive well. Moreover, the contact value of $g_{\gamma \gamma}(r)$ grows with increasing $\alpha$ 's due to the confinement of the small crystallins in the presence of the bigger $\alpha$ 's. The structural arrangements of the proteins that contribute to the different peaks are also represented by cartoons in Figure 9. Arrangements of two, three, and four small particles become more probable with increasing concentration of the large component.
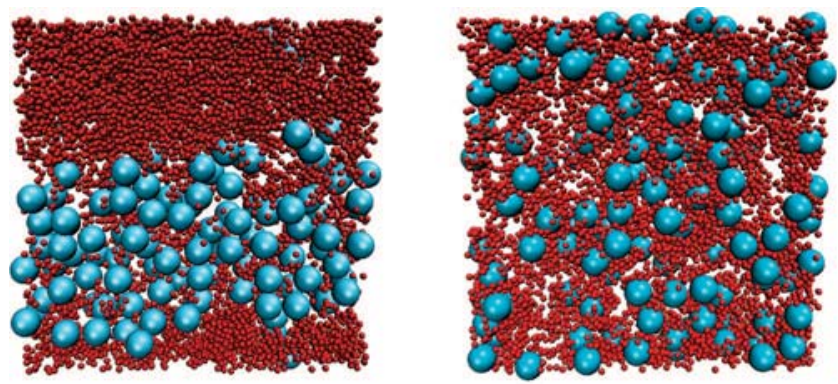

$$
\mathrm{u}_{\alpha \gamma}=0.0
$$

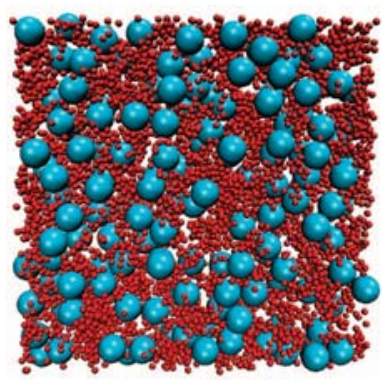

$\mathrm{u}_{\alpha \gamma}=0.55$

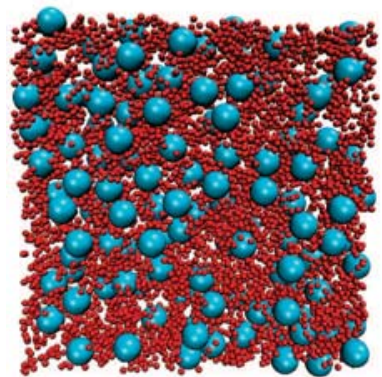

$$
\mathrm{u}_{\alpha \gamma}=1.0
$$
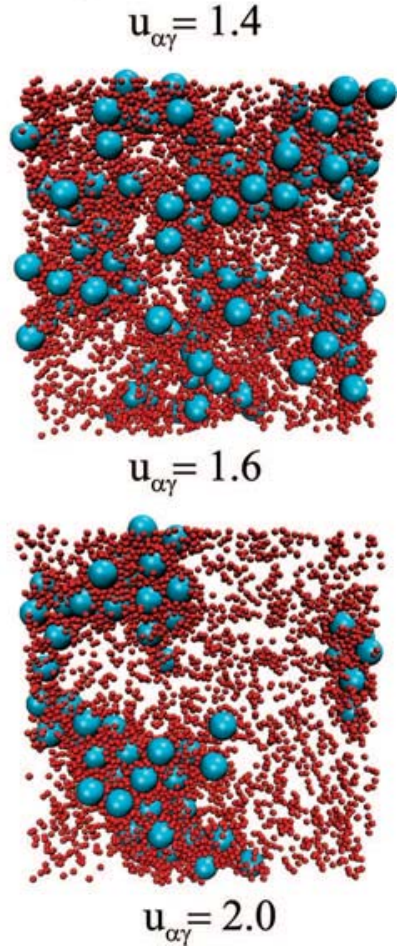

Figure 10. Molecular dynamics snapshots showing the progression from segregation of $\alpha$-crystallin and $\gamma$-crystallin by type $\left(u_{\alpha \gamma}=0\right.$, upper left), through one phase, stable mixing $\left(u_{\alpha \gamma}=0.55\right.$, second down on left), to separation of a dense phase of both proteins $\left(u_{\alpha \gamma}=2.0\right.$, lower right). Simulations performed at $\bar{T}=0.7875$.

In particular, for the interspecies $g_{\alpha \gamma}(r) \mathrm{RDFs}$, there is a sensible increase in the coordination for the case of $C_{\alpha}=0.5$ with respect to the other more critical solutions. This enforces the idea that the small proteins act as glue between the large ones and thereby inhibit demixing. With too much $\alpha-\gamma$ attraction, however, larger $\alpha-\gamma$ complexes become too stable, and strong density fluctuations start to dominate. In summary, a fine balance of the mutual attraction stabilizes the system against the two pathological situations.

4. Mixture Stability Tuning the $\alpha-\gamma$ Attraction. To study the nature of the different instabilities, a visual inspection of the simulation box can be of great help. In Figure 10, snapshots taken from simulations of the $C_{\alpha}=0.5$ mixture with different values of the mutual attraction are presented. Since the full simulation box is extremely crowded, we present only slabs of thickness equal to the diameter of the large particles. For strong and weak $u_{\alpha \gamma}$ attraction, the system is out of equilibrium (see Figure 6) and the configurations were taken while the system was still coupled to a thermostat, at the end of a long MD run.

For weak attractions, the snapshots reveal the coexistence of two different phases, one composed essentially of the smallest proteins and another where mainly the $\alpha$ are present; this is a demixing transition. Introducing an attraction between unlike proteins first counterbalances and efficiently suppresses segrega- 
tion of the two proteins into large domains. A homogeneous and stable mixture is obtained for intermediate values of the mutual attraction. As discussed before, if the attraction is further increased, the mixture becomes again unstable. Contrary to the weak attraction regime, where concentration fluctuations seemed to dominate, the density inhomogeneities are now responsible for the instability. This is evident in the snapshots for strong attractions where large regions of high and low density dominate. Thus, upon increasing the $u_{\alpha \gamma}$ parameter, one passes from a demixed state where $\alpha$-rich and $\gamma$-rich phases coexist, to a stable one-phase region, to another demixed state where a solvent-rich phase coexists with a higher density, protein-rich phase.

Transparency of concentrated $\alpha-\gamma$-crystallin mixtures is then maintained by introducing a weak, short-range attraction between $\alpha$ - and $\gamma$-crystallins, which considerably decreases the critical fluctuations due both to the attraction between $\gamma$-crystallins and the tendency of highly asymmetric mixtures to demix. As discussed before, an increase or a decrease in the shortrange attraction between unlike crystallins could have pathological effects on the lens transparency.

In this context, it is interesting to note that the existence of a mutual noncovalent attraction between $\alpha$ - and $\gamma$-crystallins has been recently demonstrated by microequilibrium dialysis experiments performed at low concentrations. ${ }^{67,68}$ Moreover, those authors found that this noncovalent attraction seems to decrease with aging of the lens. The present work confirms the existence of $\alpha-\gamma$ attraction at high densities typical of the eye lens and demonstrates molecular mechanisms by which either loss or gain of $\alpha-\gamma$ attraction could lead to phase separation and opacity in cataract disease. The former possibility is intriguing in light of the finding that $\alpha-\gamma$ attraction seems to decrease with aging. The present findings also suggest that it would now be of great interest to measure the strength of $\gamma-\alpha$ attraction in cases of known cataractogenic mutations that affect the sequence of either $\gamma$ - or $\alpha$-crystallin.

The derivation of a colloidal model of $\alpha-\gamma$ lens protein solutions, compatible with the available experimental data, opens up the possibility of performing a thermodynamic analysis of the stability of such mixtures in the full parameter space where experiments and simulations have not been performed. This is what we will discuss in the remaining part of the article.

\section{Stability of Binary Mixtures from Perturbation Theory}

Moving from one-component to binary mixtures enriches considerably the variety of possible phase behaviors. In addition to the liquid-vapor transition, the system can also exhibit demixing, and the resulting phase diagram is determined by the interplay between this two processes. ${ }^{69}$ The application of reliable liquid-state methods to determine the phase diagram of a general binary mixture is certainly more involved than the simple van der Waals analysis, and the number of free parameters is definitely larger. In order to deal with a tractable number of system parameters, the binary symmetric mixture has been considered during the past years. Important results have been obtained for these particular mixtures from the selfconsistent Ornstein-Zernike (SCOZA) approximation and the Hierarchical Reference Theory (HRT) for Yukawa fluid. ${ }^{69,70}$ However, the drawback of these methods is certainly their difficult generalization to more sophisticated mixtures.

In colloidal systems, important results have been also obtained by thermodynamic perturbation theory. Applying this theory to monodisperse short-range fluids, Gast, Hall, and Russel were able to describe the phase diagram of colloidal particles interacting by depletion interactions. ${ }^{71}$ More recently, thermodynamic perturbation theory was used to understand the interplay between phase coexistence and the glass line. ${ }^{38}$ If the perturbative approach is known to give quantitatively imprecise results near phase boundaries and near criticality, the method can be, in principle, applied to very general mixtures for which the convergence of integral-equation-based methods is still out of reach. Perturbation theory is thus well-suited to study the stability of the modeled asymmetric crystallin mixtures.

A. Perturbation Theory. We adopted the thermodynamic perturbative approach ${ }^{7,72}$ to study the thermodynamic stability of the binary mixture. The idea consists of deriving the equation of state of the interacting system by treating the attractive potential $u_{i j}(r)$ as a perturbation of the hard-sphere potential $u_{i j}^{0}(r)$. This leads to an expression for the Helmholtz free energy $F$ in terms of the averages of $\beta u_{i j}(r)$ and its powers taken over the unperturbed binary hard-sphere fluid ensemble. This reads for the first-order case

$$
\frac{F-F_{0}}{N k_{\mathrm{B}} T}=\frac{1}{2} \rho \beta \sum_{i, j=1}^{2} x_{i} x_{j} \int u_{i j}(\mathbf{r}) g_{i j}^{0}(\mathbf{r}) \mathrm{d} \mathbf{r}+O\left(\beta^{2}\right)
$$

where $F_{0}$ and $g_{i j}^{0}(r)$ are the free energy and the partial radial distribution function of the unperturbed system. A binary mixture of spherical particles of radii $d_{1}$ and $d_{2}$ is unequivocally defined by giving the number of particles of each kind, $N_{i}(i=$ $1,2)$ and the volume $V$ occupied by the system. The overall number density is then defined by $\rho=\left(N_{1}+N_{2}\right) / V$ and the mole fraction, which gives the relative concentration of the two species, by $x=N_{1} / N$.

We used the Boublik-Mansoori-Carnahan-Starling-Leland (BMCSL) equation of state for the free energy of the binary hard-sphere reference mixture $F_{0},{ }^{73-75}$ and the partial radial distribution $g_{i j}^{0}(r)$ functions were computed by solving the Ornstein-Zernike equations with the partial direct correlation functions $c_{i j}(r)$ of the binary mixture obtained by Lebowitz within the Percus-Yevick (PY) approximation. ${ }^{76}$ In order to correct the shortcomings of the PY hard-sphere distribution functions (the values at contact $g_{i j}^{0}\left(d_{i j}\right)$ and the slopes $g_{i j}^{0 \prime}\left(d_{i j}\right)$ are both too small in magnitude), we used the Grundke-Henderson procedure, a generalization to mixtures of the Verlet-Weis modifications. ${ }^{77,78}$ Details of the calculation of the $g_{i j}^{0}(r)$ 's are presented in the Appendix (see Appendix B).

The first-order term (eq 8) is evaluated exactly. Approximations for the second- and higher order terms have also been proposed. ${ }^{72,79,80}$ For one-component systems, this perturbative approach gives qualitatively good results even if, for short ranges of the attraction, it overestimates the liquid-vapor critical temperature $T_{\mathrm{c}}$ and the critical number density $\rho_{\mathrm{c}}$. Taking into account the second-order correction gives slight improvements in $\left(\rho_{\mathrm{c}}, T_{\mathrm{c}}\right)$ with respect to $\mathrm{MC}$ simulation results, but a quantitative discrepancy remains. ${ }^{47}$ Thus, the general stability picture of the binary mixture is expected not to depend on the approximations used, and a first-order approximation is certainly sufficient for the purpose of the present study.

B. Stability Criteria. The condition of thermodynamic stability of the binary mixtures is obtained by considering the Helmholtz free energy $F$ and its minima. It is convenient to scale $F$ and the other extensive variables, $V$ and $N_{i}(i=1,2)$, by the total number of particles $N=N_{1}+N_{2}$. The reduced free energy $f$ is then a function of the volume per particle $v=\rho^{-1}$ and the mole fraction $x$, as in eq 8 . The conditions of equilibrium and stability will be derived in terms of these scaled quantities. 
For equilibrium at a point on the $f-x-v$ surface, at a given temperature, it is necessary that the tangent plane lies below the surface at that point. In other words, any fluctuation in mole fraction or volume per particle from an equilibrium state should not lead the system out of this equilibrium. This condition of local stability of an equilibrium state is fulfilled if the secondorder differential of the thermodynamic potential (the Helmholtz free energy $f$ for the choice of the $v$ and $x$ variables) at constant $T$ is a positive definite quadratic form at that state

$$
\delta f^{2}=\frac{1}{2}\left[\left(\frac{\partial^{2} f}{\partial v^{2}}\right)_{T, x} \delta v^{2}+2\left(\frac{\partial^{2} f}{\partial v \partial x}\right)_{T} \delta v \delta x+\left(\frac{\partial^{2} f}{\partial x^{2}}\right)_{T, v} \delta x^{2}\right]>0
$$

This condition of stability might be rewritten introducing [f], the stiffness matrix ${ }^{81}$ of the Helmholtz free energy $f$

$$
\begin{aligned}
\delta f^{2} & =f_{v v} \delta v^{2}+2 f_{v x} \delta v \delta x+f_{x x} \delta x^{2} \\
& =(\delta v \delta x)\left(\begin{array}{ll}
f_{v v} & f_{v x} \\
f_{v x} & f_{x x}
\end{array}\right)\left(\begin{array}{l}
\delta v \\
\delta x
\end{array}\right)>0
\end{aligned}
$$

where $f_{\mu \nu} \equiv(1 / 2)\left[\left(\partial^{2} f\right) /(\partial \mu \partial v)\right]_{T}$ and $f_{\mu \mu} \equiv(1 / 2)\left[\left(\partial^{2} f\right) /\left(\partial \mu^{2}\right)\right]_{T, v}$ $(\mu, v=v, x)$. Such a quadratic form is most conveniently discussed by reduction to the diagonal form

$$
\delta f^{2}=\frac{1}{2} \sum_{k=1}^{2} \lambda_{k} \delta \xi_{k}^{2}
$$

This is achieved by a nonunique singular linear transformation of the variables which, thus, gives a different set of diagonal elements $\lambda_{\mu}$. Usually, an orthogonal transformation is used for the diagonalization of a quadratic form, but the eigenvalues obtained in this way are not as directly related to physical quantities. Thus, it is preferable to consider a different, nonorthogonal transformation which allows one to relate the eigenvalues of the quadratic form to physical meaningful quantities. ${ }^{81,82}$

Factorizing with respect to $f_{x x}$ and completing the square in eq 9 yields

$$
\begin{aligned}
\delta f^{2} & =\left(f_{v v}-\frac{f_{v x}^{2}}{f_{x x}}\right) \delta v^{2}+f_{x x}\left(\delta x+\frac{f_{v x}}{f_{x x}}\right)^{2} \\
& =\left(f_{v v}-\frac{f_{v x}^{2}}{f_{x x}}\right) \delta \xi_{2}^{2}+f_{x x} \delta \xi_{1}^{2}
\end{aligned}
$$

where

$$
\begin{aligned}
& \delta \xi_{1}=\left(\delta x+\frac{f_{v x}}{f_{x x}} \delta v\right) \\
& \delta \xi_{2}=\delta v
\end{aligned}
$$

The conditions for a system to be thermodynamically stable (or metastable) can thus be written either

$$
f_{x x}>0 \quad \text { and } \quad f_{v v}-\frac{f_{v x}^{2}}{f_{x x}}>0
$$

or

$$
f_{v v}>0 \quad \text { and } \quad f_{x x}-\frac{f_{v x}^{2}}{f_{v v}}>0
$$

when computing the factorization with respect to $f_{v v}$.

The second-order derivatives of a scaled thermodynamic potential with respect to a scaled extensive variable must be positive for a system to be stable. Moreover, even if the transformations considered take place at constant mole fraction and volume per particle, it is possible to consider slightly different thermodynamic potentials and express the stability criteria in terms of their derivatives, in which all variables held constant are intensive variables. ${ }^{82}$ Indeed, instead of the Helmholtz free energy, one might consider the Gibbs free energy $g$ or the quantity $f^{\prime} \equiv f-x\left(\mu_{2}-\mu_{1}\right)$ (with $\mu_{i}$ as the chemical potentiel of species $i$ ) and express the condition of stability in terms of the partial derivatives of these potentials

$$
\begin{aligned}
\left(\frac{\partial^{2} f^{\prime}}{\partial v^{2}}\right)_{T, \mu_{2}-\mu_{1}} & =\left(\frac{\partial^{2} f}{\partial v^{2}}\right)_{T, x}-\left(\frac{\partial^{2} f}{\partial v \partial x}\right)_{T}\left(\frac{\partial^{2} f}{\partial x^{2}}\right)_{T, v}^{-1} \\
& \equiv f_{v v}-\frac{f_{v x}^{2}}{f_{x x}} \\
\left(\frac{\partial^{2} g}{\partial x^{2}}\right)_{T, P} & =\left(\frac{\partial^{2} f}{\partial x^{2}}\right)_{T, v}-\left(\frac{\partial^{2} f}{\partial v \partial x}\right)_{T}\left(\frac{\partial^{2} f}{\partial v^{2}}\right)_{T, x}^{-1} \\
& \equiv f_{x x}-\frac{f_{v x}^{2}}{f_{v v}}
\end{aligned}
$$

The stability criteria previously derived require these two quantities to be positive. An interesting point is that the derivatives in which all variables held constant are intensive, and which are usually named primary stability indicators, are always less than or equal to corresponding derivatives with some extensive variables held constant (secondary indicators). In onecomponent systems, the inverse of the isothermal compressibility $\chi_{T}^{-1} \equiv\left(\partial^{2} f / \partial v^{2}\right)_{T}$ is a primary indicator and vanishes as the instability is reached. This instability, called mechanical instability, occurs when density fluctuations become infinite in the system and allows the nucleation of low and high density phases that then coexist. This instability is responsible for the usual liquid-vapor phase transition encoutered in one-component systems.

In binary mixtures, the system might become mechanically instable without the inverse of the corresponding compressibility $\chi_{T, x}^{-1} \equiv\left(\partial^{2} f / \partial v^{2}\right)_{T, x}$ vanishing since it follows that all that is required is, instead, that $\chi_{T, \mu_{2}-\mu_{1}}^{-1} \equiv\left(\partial^{2} f^{\prime} / \partial v^{2}\right)_{T, \mu_{2}-\mu_{1}}$ goes to zero. In other words, it should be remembered that the isothermal compressibility, the mechanical stability indicator in a onecomponent mixture, is no longer a unique quantity in binary mixtures. Besides mechanical instability, strong concentration fluctuations can lead to demixing, that is, a separation of the system into phases of different concentrations. In this case, $\left(\partial^{2} f\right)$ $\left.\partial x^{2}\right)_{T, v}$ is a material stability indicator that diverges as the instability boundary is reached, and the corresponding primary stability indicator is provided by $\left(\partial^{2} g / \partial x^{2}\right)_{T, P}$. Except in special cases, both mechanical and material instabilities will, in general, appear simultaneously.

C. Results. The experimental work provided a first picture of the large-scale fluctuations in this binary system. We want now to study the loci where these fluctuations become un- 


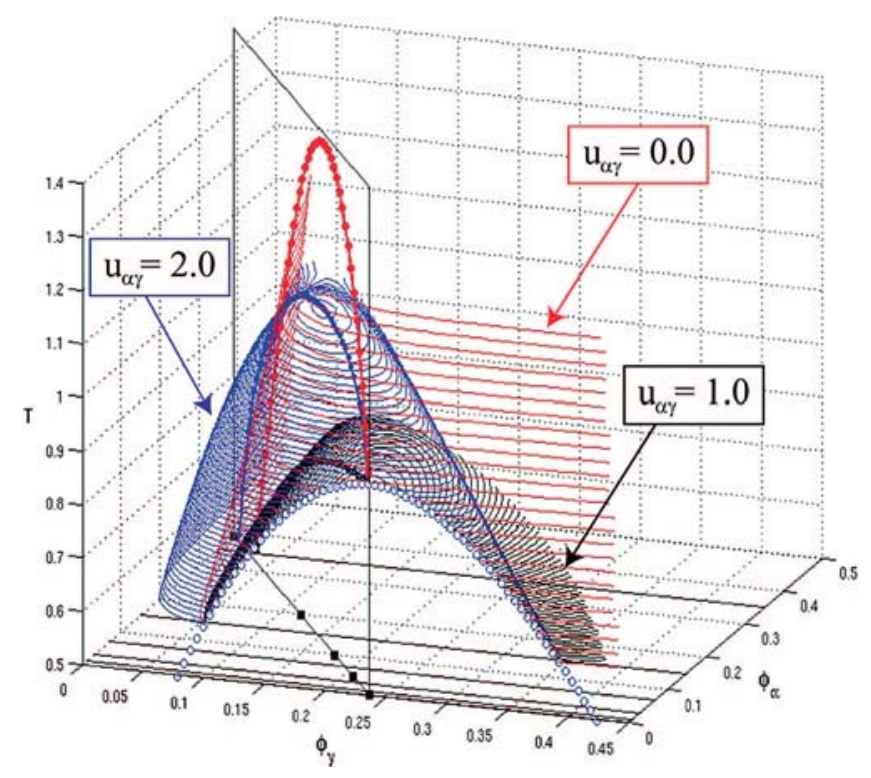

Figure 11. Attraction between $\alpha$ and $\gamma$ lens crystallins affects the thermodynamic stability of their concentrated mixtures in a nonmonotonic fashion. The red spinodal surface shows the very highly elevated spinodal for hard-sphere interactions between $\alpha$ and $\gamma$, while the black and blue surfaces show, respectively, the suppressed and then reelevated surfaces for square-well depths of 1 and 2. The spinodal of the pure $\gamma$ mixture is also drawn (open dots). The lines on the bottom of the figure indicate $\phi_{\alpha}$ values for the 2D cuts of the stability boundary surface presented in Figure 12. The dark squares correspond to the mixing ratios $C_{\alpha}$ of the mixtures on which the SANS experiments were performed.

bounded, that is, the spinodal surface, by the perturbative model introduce by eq 8 . The instability or spinodal surface indicates the temperature $T^{*}$ below which the binary mixture cannot exist as a homogeneous phase. Indeed, upon crossing the spinodal, it is thermodynamically more favorable for the system to split in two or more coexisting phases. The spinodal surface provides an indication of the coexistence surface (the binodal) since the latter will be located at slightly higher temperature, except for critical lines, where the binodal and spinodal merge.

The scattering intensities can be used as a qualitative estimate of the distance to the unstable region of the binary mixture; the pure $\gamma$ solution is already highly critical, being just above the critical point. With addition of $\alpha$ 's, keeping the temperature constant, the scattering intensity increases first $\left(C_{\alpha}=0.12,0.25\right)$, but then, fluctuations are suppressed by further addition of $\alpha$ 's (see Figure 1). Quite consistent with previous findings, ${ }^{28}$ these data suggest that, upon increasing the $\alpha$ concentration, the system first get closer to the instability boundary and subsequently moves away from it. From the simulations, it appears, however, that this behavior depends strongly on the value of the mutual attraction (see Figure 6). It is important to test whether our perturbative scheme can reproduce this behavior. If this is achieved, we will be able to study the stability of the system in the whole parameter space, something that cannot be achieved by experiments and MD simulations.

1. 3D Spinodal Surface. We shall present our results in the $\left(\phi_{\alpha}-\phi_{\gamma}-T\right)$ space, where $\phi_{\alpha}$ and $\phi_{\gamma}$ are the partial packing fractions defined as $\phi_{\mu}=(\pi / 6) d_{\mu}^{3} \rho x(\mu=\alpha, \gamma)$. This is the best way to represent graphically the spinodal surface due to the strong size asymmetry of the mixture that we are studying. In Figure 11, we present the spinodal surfaces of three different mixtures, corresponding to three values of the $u_{\alpha \gamma}$ attraction, $u_{\alpha \gamma}=0,1$, and 2 . In order to compute the instability surface according to eq 9, we computed the derivatives of the Helmholtz

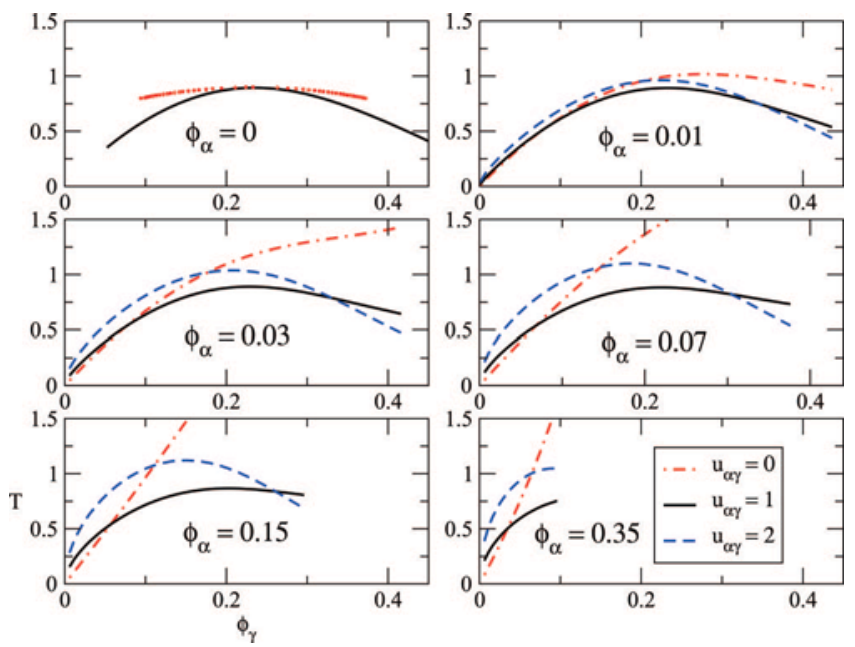

Figure 12. 2D cut at constant $\phi_{\alpha}$ of the instability surfaces for different values of the interaction $u_{\alpha \gamma}=0,1$, and 2 . The values of $\phi_{\alpha}$ are indicated in each panel. The upper left panel shows the coexistence curve of the one-component $\gamma$ mixture (dots) and the spinodal lines of the three mixtures are superimposed.

free energy by finite differences up to seven points. To better visualize the shape of the spinodal surfaces, in Figure 12, we report several cuts at constant $\phi_{\alpha}$ of the full diagram (Figure 11).

Before the binary mixtures, we first consider the spinodal of the pure $\gamma$ mixture, that is, a mixture of square-well particles with a range of $\lambda_{\gamma \gamma}=0.25 d_{\gamma}$. This is represented by dots in Figure 11. In this one-component limit, the condition of stability simplifies to $\left(\partial^{2} f / \partial v^{2}\right)_{T}>0$. The coexistence curve is also determined (Figure 12, first panel). We find a critical packing fraction of $\phi_{\gamma}^{\mathrm{c}_{\mathrm{PT}}}=0.236$ and $T_{\gamma}^{\mathrm{c}_{\mathrm{PT}}}=0.895$, values that are in agreement with previous studies. ${ }^{47,83}$ Indeed, it has been shown that perturbation theory slightly overestimates $T^{\mathrm{c}}$ and the corresponding critical density.

As expected, by gradually increasing $\phi_{\alpha}$, the spinodal surface always develops starting from the pure $\gamma$ spinodal curve $\left(\phi_{\alpha}=\right.$ $0)$. As we shall see, the shape of the spinodal will depend strongly on the value of the mutual attraction between unlike species. The peculiarity of the spinodal for the purely repulsive $\left(u_{\alpha \gamma}=0\right)$ mixture, presented in Figure 11, is the strong enhancement of $T^{*}$ upon increasing, even only slightly, $\phi_{\alpha}$. When this happens, the stability boundary moves to higher temperatures. In particular, it appears clearly from the 2D cuts presented in Figure 12 that the destabilization is much more pronounced at high $\phi_{\gamma}$. This seems to support the idea that, for the repulsive case, the instability is caused by the depletion interaction that was first encountered in asymmetric hard-sphere mixtures with size ratios larger than 1:5.6,84 Depletion is an effective attraction of purely entropic origin between the larger components in binary mixtures with a strong size asymmetry. In particular, the strength of the attraction increases with the density of the small components and can even induce demixing between the two species. Indeed, we observed this kind of demixing in the MD simulations for this value of the interaction (see Figure 10).

Following the same line of reasoning as that for the MD simulations, we introduced an interspecies attraction $u_{\alpha \gamma}$ to stabilize the mixture. The spinodal of a mixture with $u_{\alpha \gamma}=1$ is represented in Figure 11. This value of the attraction was found to be the one that most lowered the location of the stability boundary. The mixture remains stable at temperatures equivalent to the ones found for the pure $\gamma$ mixture. The behavior of the 


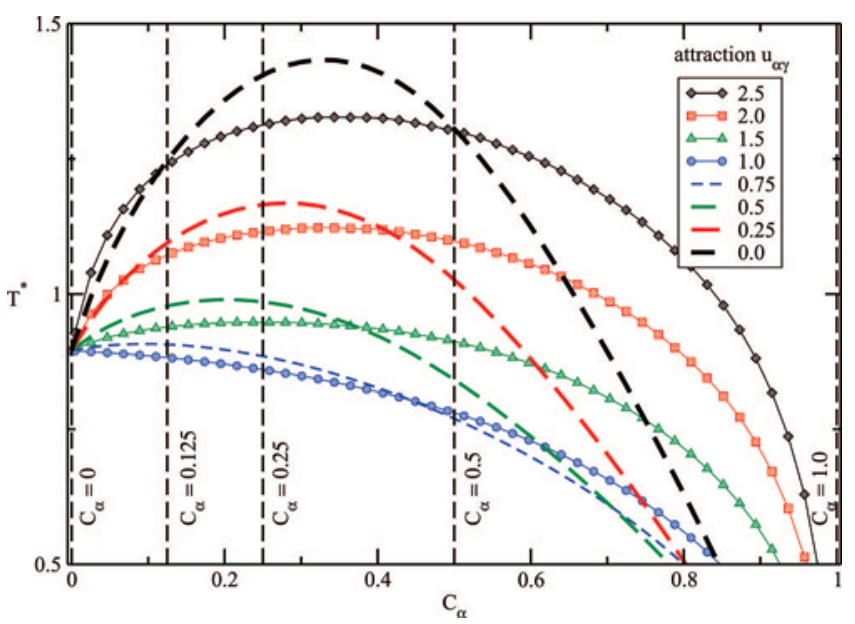

Figure 13. 2D section of the instability surface on the experimental $\bar{\phi}_{\alpha}-\phi_{\gamma}^{\mathrm{cPT}}$ line drawn in Figure 11 . The temperature $T^{*}$ at which the mixtures become unstable is presented as a function of the relative mixing ratio $C_{\alpha}$ for values of $u_{\alpha \gamma}$ ranging from the hard-sphere limit up to 3 .

spinodal appears to be quite independent of the concentration of the larger components (see Figure 12). Thus, the attraction efficiently counterbalances the depletion attraction between the large components, and the consequent demixing is reduced.

Upon further increasing the attraction, the spinodal surface moves again to higher temperatures but in a different way than that for the hard-sphere case. The occurrence of a very localized maximum at intermediate values of $\phi_{\alpha}$ and $\phi_{\gamma}$ is observed in Figure 11 for the instability surface of a mixture with $u_{\alpha \gamma}=2$. Interestingly, the mixture is also more critical than the previous two at low $\phi_{\gamma}$, while no enhancement of the instability at high $\phi_{\gamma}$ is found (Figure 12). This is not surprising since a strong interspecies attraction induces mechanical instability. In this case, the system tends to split into regions of very different density, similar to what is observed for the one-component attractive case. At low density, this tendency will be promoted due to the free space available, while at high density, the mixture is already highly packed, and no additional effect will be observed.

2. Spinodals on the Experimental Plane. The SANS experiments have been performed by mixing different amounts of a pure $\alpha$ solution $\left(\bar{\phi}_{\alpha}=0.391\right)$ and a pure $\gamma$ solution $\left(\bar{\phi}_{\gamma}=0.183\right)$. The different mixing ratios $C_{\alpha}=0,0.125,0.25,0.5$, and 1 are thus distributed along a line on the $\phi_{\alpha}-\phi_{\gamma}$ plane. In order to test the validity of our method, we investigate the instability with more attention to this cut of the parameter space represented by a plane in Figure 11. As already discussed, perturbation theory tends to overestimate the location of the critical point.

Then, to be consistent with the fact that the experiments took place near the pure $\gamma$ critical point, we consider the pure $\gamma$ solution to be at $\phi_{\gamma}^{\mathrm{c}_{\mathrm{pT}}}=0.236$, that is, the critical packing fraction as obtained from the theory, instead of $\bar{\phi}_{\gamma}$. In this way, we are sure that the perturbation model for the pure $\gamma$ solution is indeed at the critical density.

The instability boundaries on this particular cut of the parameter space are presented in Figure 13 for different values of the $u_{\alpha \gamma}$ attraction. A specific trend emerges. With no or low interspecies attraction, the spinodal lines move quickly to high temperature upon increasing the concentration of $\alpha$ 's. This tendency is suppressed for intermediate values of the attraction, and for $u_{\alpha \gamma}=1$, the spinodal temperatures of the mixtures are

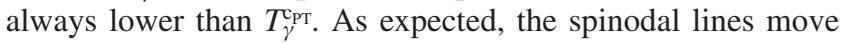
again to higher temperature for stronger interactions.

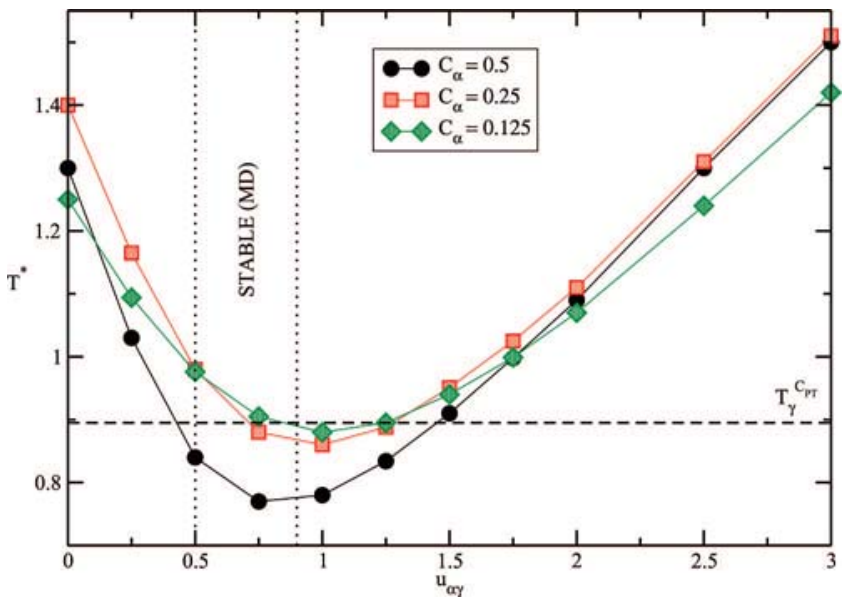

Figure 14. Temperature of instability $T^{*}$ as a function of the interaction strength $u_{\alpha \gamma}$ for the mixing ratios $C_{\alpha}=0.125,0.25$, and 0.5 (dashed line in Figure 13). The stable region obtained from the MD simulations for the $C_{\alpha}=0.5$ case and the critical temperature of the pure $\gamma$ mixture (dashed line) are also drawn.

From these calculations, we notice that with an attraction of around $u_{\alpha \gamma}=0.5$, the best description of the experimental behavior is obtained, that is, a maximum of $T^{*}$ between $C_{\alpha}=$ 0.125 and 0.25 and a decrease of the spinodal temperature for the $C_{\alpha}=0.5$ case, in very good agreement with the shape of the phase boundaries found previously for $\alpha-\gamma$ mixtures. ${ }^{28}$ This is also in agreement with the results from the MD simulations where an attraction of $u_{\alpha \gamma}=0.55$ gives the best agreement with the experimental data. With weaker attraction, a maximum of the instability temperature $T^{*}$ is obtained for $C_{\alpha}=0.35$, and the mixture is still unstable for $C_{\alpha}=0.5$. Increasing the interaction makes the mixture highly unstable for all $C_{\alpha}=0.125$, 0.25 , and 0.5 , and $T^{*}$ is larger than $T_{\gamma}^{\mathrm{c}_{\mathrm{p}}}$ for mixing ratios much beyond $C_{\alpha}=0.5$.

With this theoretical approach, we can extract the spinodal temperature $T^{*}$. Since the scattering intensity at low $q$ will diverge when approaching the instability boundary, we report $T^{*}$ as a function of the $u_{\alpha \gamma}$ attraction for the different mixing ratios (Figure 14) and confront it with the results of the MD simulations for the $C_{\alpha}=0.5$ mixture, for which $I\left(q^{*}=\right.$ $0.0467 \mathrm{~nm}^{-1}$ ) as a function of $u_{\alpha \gamma}$ was plotted (Figure 6). The same nonmonotonic behavior as that observed in the MD simulations is recovered for all three mixtures. Moreover, the stability region that emerges from the simulations is in good correspondence with the minimum of $T^{*}$ with respect to $u_{\alpha \gamma}$. From what we discussed above, it can be argued that while perturbation theory gives only a qualitative agreement for the location of the spinodal temperature and critical densities, it provides the right value of the interspecies attraction to reproduce the stabilization effect observed in the experiments and the simulations.

Our model gives good results for the spinodal of the binary mixtures and opens the possibility to explore the full phase diagrams in the future. In this sense, a special property of the $\alpha-\gamma$ mixture is that their phase separation process involves substantial segregation by protein type, as well as by concentration. ${ }^{28}$ The experimental liquid-liquid phase separation tie lines indicates the coexistence of dilute phases, rich in $\alpha$-crystallins, and dense phases with predominance of $\gamma$-crystallins. This gives rise to the tilted tie line pairs reported in Figure 15 and taken from the experiments reported in ref 28. In this work, we limit our investigation to the spinodal stability of the mixtures. The spinodal is always located below the coexistence surface and 


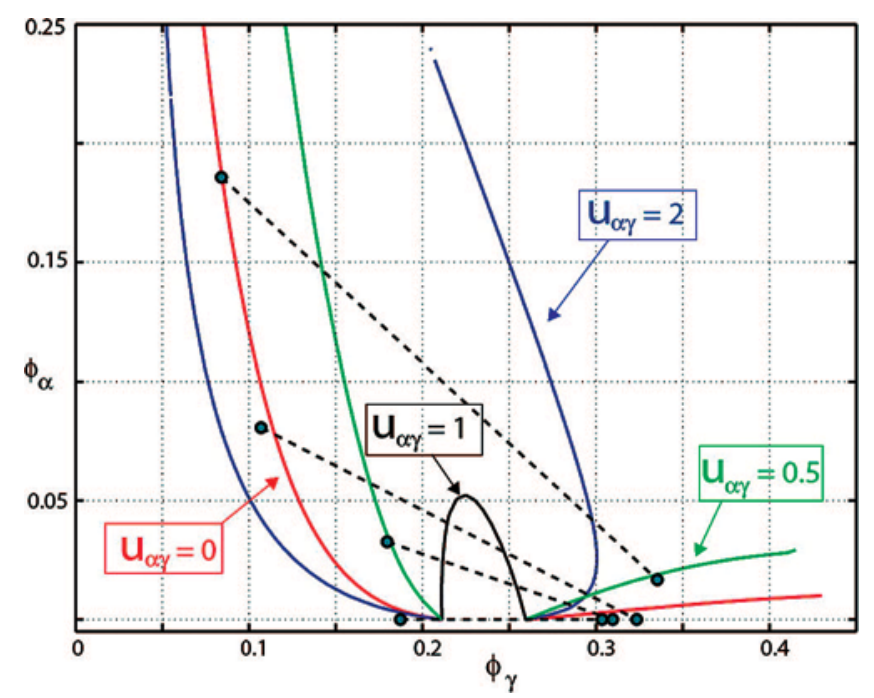

Figure 15. 2D cut of the spinodal surface at $T=0.98 T_{\gamma}^{\mathrm{c}_{\mathrm{pT}}}$ for $u_{\alpha \gamma}=$ $0,0.5,1$, and 2 . The experimental tie line pairs (full circles and dashed lines) were taken from ref 28.

can give a valid indication of the shape of the tie lines. Cuts of the spinodal surface at a temperature slightly below the critical temperature of the pure $\gamma$ mixture are presented in Figure 15. The perturbative spinodal surface with weak attraction is indeed compatible with the scenario of these extremely tilted experimental tie lines (see, in particular, the $u_{\alpha \gamma}=0.5$ mixture). This represents a further confirmation of the validity of the approach based on thermodynamic perturbation theory.

\section{Conclusions}

We have developed a colloidal model of $\alpha$ - and $\gamma$-crystallin proteins that is able to account for the experimental spectra of the pure solutions and the mixtures of these proteins. Very good agreement on the length scale important for the lens transparency was obtained. We found that the transparency of concentrated crystallin mixtures is maintained by introducing a weak, shortrange attraction between $\alpha$ - and $\gamma$-crystallins. This attraction considerably decreases the critical fluctuations due to the attraction between $\gamma$-crystallins and the tendency of a highly asymmetric mixture to demix.

The presence of a net attraction between $\alpha$ - and $\gamma$-crystallin has been recently found in experiments performed at low concentration. ${ }^{67,68,85}$ Our work gives evidence for $\alpha-\gamma$ attraction at high protein concentrations typical of the eye lens and suggests that this attraction may play a key role for the stability of eye lens cytoplasm with respect to phase separation. Thus, changes in the magnitude of the $\alpha-\gamma$ attraction might contribute to some forms of cataract. Due in part to its small magnitude, the molecular origin of the inferred net short-range attraction between unlike crystallins remains a challenging and open question, as it is for short-range attraction of many globular proteins including $\gamma$-crystallins and lysozyme., ${ }^{4,55}$

The second part of the paper was dedicated to the calculation of the stability boundary of the binary mixture in the full parameter space via a thermodynamic perturbation theory approach. Perturbation theory turns out to be well-suited to study the instability of asymmetric binary square-well mixtures. All of the critical behavior expected from the SANS experiments was correctly recovered, at least qualitatively. We also obtained quantitative agreement with the results of the MD simulations for the determination of the strength of the mutual attraction. This theoretical approach allowed us to confirm the validity of
TABLE 2: Parameters of the Molecular Dynamics Simulations for the Different $C_{\alpha}$ Mixtures $^{a}$

\begin{tabular}{lccr}
\hline$C_{\alpha}$ & $\phi_{\alpha}$ & $\phi_{\gamma}$ & \multicolumn{1}{c}{$N_{\alpha}$} \\
\hline 0 & & 0.184 & \\
0.125 & 0.049 & 0.163 & 104 \\
0.25 & 0.098 & 0.140 & 240 \\
0.5 & 0.195 & 0.093 & 704 \\
1 & 0.391 & & 32000
\end{tabular}

${ }^{a}$ The packing fraction of each component $\phi_{\alpha}$ and $\phi_{\gamma}$ and the number of $\alpha$ components $N_{\alpha}$ are given (with $N_{\alpha}+N_{\gamma}=32000$ ).

the model that we built for the mixture of crystallin proteins from a totally different route. The calculation of the spinodal surface in the full parameter space gave also a better insight into the different mechanisms responsible for the instability in the binary mixture. Following the encouraging results obtained for the instability boundary, efforts will be made toward the determination of the full phase coexistence using the same perturbative scheme.

The present work recognizes two new molecular mechanisms by which altered eye lens crystallin protein interactions can potentially lead to cataract disease. First, concentrated mixtures of $\alpha$ - and $\gamma$-crystallin become unstable with respect to proteincomposition-type phase separation if there is too little $\alpha-\gamma$ attraction. On the other hand, too much $\alpha-\gamma$ attraction makes concentrated $\alpha-\gamma$ mixtures unstable with respect to proteinconcentration-type phase separation. Moreover, in combination with recent work, ${ }^{28}$ the present work further underlines the role of size disparity between $\alpha$ - and $\gamma$-crystallins in helping to produce phase separation of concentrated $\alpha-\gamma$ mixtures, which is dramatically enhanced in comparison with that of the component proteins.

It is very important to recognize that each of these crystallin instability mechanisms can potentially contribute to cataract at temperatures well above those at which thermodynamic instability occurs. First, as has been shown near the phase separation of $\gamma$-crystallins alone, ${ }^{22,26-28}$ each transition will dramatically increase light scattering at temperatures well above phase separation, in the single-phase region of the phase diagram. Second, the very reason for enhanced light scattering, namely, that proximity to phase separation enhances spontaneous local concentration fluctuations, can also serve to accelerate nucleation and growth of protein aggregates. ${ }^{86}$

It is clearly important to identify specific molecular factors that mediate both the strength of $\gamma-\alpha$ attraction and the size disparity between these proteins and to identify the effects of shape and orientation-dependent interactions, not considered here, on $\gamma-\alpha$ mixture instability.

More generally, this work has explored one out of the three principal concentrated binary lens crystallin mixtures, each of which can serve as a baseline for effective study of realistically concentrated $\gamma-, \alpha-$, and $\beta$-crystallin mixtures. As such, it represents a step toward the needed understanding of the chemically specific origins of short-range order and eye lens transparency.

Acknowledgment. We thank A. Baldereschi for support and useful discussion and C. Likos for interesting comments. The SANS experiments were performed at the Swiss spallation neutron source SINQ, Paul Scherrer Institute, Villigen, Switzerland. We are grateful for the neutron beam time, and we acknowledge the help of our local contact J. Kohlbrecher. This work was supported by the Swiss National Science Foundation (Grant No. 99200021-105382/1, 200020-109499, and 200020- 
117755 and No. PP0022119006), the State Secretariat for Education and Research (SER) of Switzerland, the Marie Curie Network on Dynamical Arrest of Soft Matter and Colloids (No. MCRTN-CT-2003504712), and NIH (U.S.) Grant No. EY11840 and EY018249-01.

\section{Appendix}

A. Resolution Function. In order to account for the experimental smearing, we derived a general resolution function resulting from the combined effect of wavelength spread $(w)$, finite collimation $(c)$, and detector resolution $(d)$. The resolution function $R(\mathbf{q},\langle\mathbf{q}\rangle)$ describes the distribution of the radiation with scattering vector $\mathbf{q}$ contributing to the scattering for the setting $\langle\mathbf{q}\rangle$. The measured intensity at $\langle\mathbf{q}\rangle$ is then proportional to

$$
I(\langle\mathbf{q}\rangle)=\int R(\mathbf{q},\langle\mathbf{q}\rangle) \frac{\mathrm{d} \sigma(\mathbf{q})}{\mathrm{d} \mathbf{\Omega}} \mathrm{d} \mathbf{q}
$$

where $\mathrm{d} \sigma(\mathbf{q}) / \mathrm{d} \boldsymbol{\Omega}$ is the scattering cross section. We followed the method proposed by Pedersen et $a l .{ }^{45}$ in which the different contributions to the resolution function are all approximated by Gaussian functions and are derived separately for each contribution, assuming the other ones to be negligible

$$
R(\langle\boldsymbol{q}\rangle)=\frac{1}{\sqrt{\left(2 \pi \sigma^{2}\right)}} \mathrm{e}^{-(q-\langle q\rangle)^{2} / 2 \sigma^{2}}
$$

The Gaussian functions are determined by calculating the width $\sigma$ of the distribution and are defined in a way to have the same full-width at half-maximum (FWHM) value as the distribution they approximate. In the following, $\Delta \lambda$ will denote the FWHM of the wavelength distribution and $\Delta$ the FWHM of the distribution of $(s-\langle s\rangle)$, where $\langle s\rangle$ is the distance from the beam center at the detector to the point on the detector with a scattering angle of $\langle 2 \theta\rangle$. The combined resolution function due to all three contributions is then calculated assuming them to be independent and can be approximated by a Gaussian with a width $\sigma$ given by $\sigma^{2}=\sigma_{c}^{2}+\sigma_{d}^{2}+\sigma_{w}^{2}$ where

$$
\begin{gathered}
\sigma_{c}=\langle k\rangle \cos \langle\theta\rangle \frac{\Delta \beta_{1}}{2(2 \ln (2))^{2}} \\
\sigma_{d}=\langle k\rangle \cos \langle\theta\rangle \cos ^{2}\langle 2 \theta\rangle \frac{\Delta}{2 l(2 \ln (2))^{2}} \\
\sigma_{w}=q_{0} \frac{\Delta \lambda}{\langle\lambda\rangle} \frac{1}{2(2 \ln (2))^{1 / 2}}
\end{gathered}
$$

with $\theta=\arcsin (\lambda q / 2 \pi)$, with wavelength $\lambda$ for $q$ vector $q$, with $R_{1}$ being the radius of the source aperture, $r_{2}$ the radius of the sample aperture, $L$ the source-sample distance, $l$ the detectorsample distance, and defining $a_{1}=r_{1}\left(L+l / \cos ^{2}(2 \theta)\right)^{-1}, a_{2}=$ $r_{2} \cos ^{2}(2 \theta)$, and

$$
\begin{aligned}
\Delta \beta_{1}= & 2 \frac{r_{1}}{L}-\frac{1}{2} \frac{r_{2}^{2}}{r_{1}} \frac{\cos ^{2}\langle 2 \theta\rangle}{l^{2} L}\left(L+\frac{l}{\cos \langle 2 \theta\rangle}\right)^{2} \quad a_{1}>a_{2} \\
\Delta \beta_{1}= & 2 r_{2}\left(\frac{1}{L}+\frac{\cos ^{2}\langle 2 \theta\rangle}{l}\right)- \\
& \frac{1}{2} \frac{r_{1}^{2}}{r_{2}} \frac{l}{L} \frac{1}{\cos ^{2}\langle 2 \theta\rangle\left(L+\frac{l}{\cos ^{2}\langle 2 \theta\rangle}\right)} \quad a_{1}<a_{2}
\end{aligned}
$$

When the scattering pattern is circularly symmetric, the twodimensional integration involved in the convolution of the cross section and the resolution function can be replaced by a onedimensional integration

$$
I(\langle q\rangle)=\int_{0}^{\infty} R_{\mathrm{av}}(q,\langle q\rangle) \frac{\mathrm{d} \sigma(q)}{\mathrm{d} \Omega} \mathrm{d} q
$$

with the averaged resolution function $R_{\mathrm{av}}(q,\langle q\rangle)$

$$
\begin{aligned}
R_{\mathrm{av}}(q,\langle q\rangle) & =\int_{-\pi}^{\pi} R(q,\langle q\rangle) q \mathrm{~d} \phi \\
& =\frac{q}{\sigma^{2}} \mathrm{e}^{-\left(q^{2}-\langle q\rangle^{2}\right) / 2 \sigma^{2}} I_{0}\left(q\langle q\rangle \sigma^{-2}\right)
\end{aligned}
$$

in which $I_{0}$ is the modified Bessel function of first kind and zeroth order and $\sigma=\left(\sigma_{c}^{2}+\sigma_{d}^{2}+\sigma_{w}^{2}\right)^{1 / 2}$.

B. Calculation of $\boldsymbol{g}_{i j}(\boldsymbol{r})$. Perturbation theory relies on an accurate description of the reference system, for which we have chosen a binary mixture of hard-sphere particles. In particular, the partial radial distribution functions $g_{i j}$ that appear in the expression for the Helmholtz free energy must be evaluated. The Ornstein-Zernike (OZ) integral equations solved with the Percus-Yevick (PY) closure are often used to supply reference approximations for the $g_{i j}$ 's.

1. Ornstein-Zernike Equations with Percus-Yevick Closure for Binary Hard-Sphere Mixtures. The OZ equation is an integral equation that was first introduced by L. S. Ornstein and F. Zernike in their investigation of the density fluctuations near the critical point. It relates the total correlation function $h(\mathbf{r})=g(\mathbf{r})-1$ to the direct correlation function $c(\mathbf{r}) .{ }^{7}$ For a one-component system, homogeneous and isotropic, the $\mathrm{OZ}$ relation can be written as

$$
h(\mathbf{r})=c(\mathbf{r})+\rho \int c\left(\left|\mathbf{r}-\mathbf{r}^{\prime}\right|\right) h\left(\mathbf{r}^{\prime}\right) \mathrm{d} \mathbf{r}^{\prime}
$$

This expression, which can be solved recursively, describes the fact that the total spatial correlation is due, in part, to the direct correlation but also to the indirect correlation propagated via an increasingly large number of intermediate positions. From the analysis of graphical expansions, ${ }^{7}$ it is very likely that the range of $c(\mathbf{r})$ is comparable with the range of the pair potential $v(\mathbf{r})$, while, because of the effects of the indirect correlation, $h(\mathbf{r})$ can be much longer ranged, especially in the vicinity of a critical point.

The Fourier transform of the $\mathrm{OZ}$ equation yields

$$
h(\mathbf{k})=c(\mathbf{k})+\rho h(\mathbf{k}) c(\mathbf{k})
$$

which gives an algebraic relation between $h(\mathbf{k})$ and $c(\mathbf{k})$ 


$$
h(\mathbf{k})=\frac{c(\mathbf{k})}{1+\rho c(\mathbf{k})}
$$

For homogeneous and isotropic multicomponent systems, the Fourier transform of the $\mathrm{OZ}$ relations becomes

$$
h_{\alpha \beta}(\mathbf{k})=c_{\alpha \beta}(\mathbf{k})+\sum_{\gamma} \rho_{\gamma} c_{\alpha \gamma}(\mathbf{k}) h_{\beta \gamma}(\mathbf{k})
$$

For a binary mixtures of species 1 and 2, the following system must be solved

$$
\begin{aligned}
& h_{11}(\mathbf{k})=c_{11}(\mathbf{k})+\rho_{1} c_{11}(\mathbf{k}) h_{11}(\mathbf{k})+\rho_{2} c_{12}(\mathbf{k}) h_{21}(\mathbf{k}) \\
& h_{22}(\mathbf{k})=c_{22}(\mathbf{k})+\rho_{1} c_{21}(\mathbf{k}) h_{12}(\mathbf{k})+\rho_{2} c_{22}(\mathbf{k}) h_{22}(\mathbf{k})(29) \\
& h_{12}(\mathbf{k})=c_{12}(\mathbf{k})+\rho_{1} c_{11}(\mathbf{k}) h_{12}(\mathbf{k})+\rho_{2} c_{12}(\mathbf{k}) h_{22}(\mathbf{k})
\end{aligned}
$$

with $c_{\alpha, \gamma}(\mathbf{k})=c_{\gamma, \alpha}(\mathbf{k})$ and $h_{\alpha, \gamma}(\mathbf{k})=h_{\gamma, \alpha}(\mathbf{k})$. In order to solve this set of linear equations, we define

$$
\begin{aligned}
& R_{1}(\mathbf{k})=\rho_{1} c_{11}(\mathbf{k})-1 \\
& R_{2}(\mathbf{k})=\rho_{2} c_{12}(\mathbf{k}) \\
& R_{3}(\mathbf{k})=\rho_{1} c_{12}(\mathbf{k}) \\
& R_{4}(\mathbf{k})=\rho_{2} c_{22}(\mathbf{k})-1
\end{aligned}
$$

and

$$
\begin{aligned}
& S_{1}(\mathbf{k})=-c_{12}(\mathbf{k})+\frac{R_{1}(\mathbf{k})}{R_{3}(\mathbf{k})} c_{22}(\mathbf{k}) \\
& S_{2}(\mathbf{k})=-c_{22}(\mathbf{k})-\frac{R_{3}(\mathbf{k}) R_{4}(\mathbf{k})}{R_{2}(\mathbf{k}) R_{3}(\mathbf{k})-R_{1}(\mathbf{k}) R_{4}(\mathbf{k})} S_{1}(\mathbf{k})
\end{aligned}
$$

The solution of OZ equations can be cast

$$
\begin{aligned}
& h_{11}(\mathbf{k})=\frac{1}{R_{1}(\mathbf{k})}\left[-c_{11}(\mathbf{k})-\frac{R_{2}(\mathbf{k})}{R_{3}(\mathbf{k})} S_{2}(\mathbf{k})\right] \\
& h_{12}(\mathbf{k})=\frac{1}{R_{3}(\mathbf{k})} S_{2}(\mathbf{k}) \\
& h_{11}(\mathbf{k})=\frac{R_{3}(\mathbf{k})}{R_{2}(\mathbf{k})-R_{1}(\mathbf{k}) R_{4}(\mathbf{k})} S_{1}(\mathbf{k})
\end{aligned}
$$

In order to calculate $h_{i j}(k)$, the partial direct correlation functions $c_{i j}(k)$ must be determined. The $c_{i j}(r)$ 's of binary hard-sphere mixtures have been obtained analytically by Lebowitz within the PY approximation (relations 37 to 40 in ref 76). Starting from these expressions of $c_{i j}(r)$, we calculated their Fourier transform $(\mathrm{FT}) c_{i j}(k)$, which is still analytical, and then solved the previous relations.

The last step for determining the radial distribution function of binary hard-sphere mixtures involves the calculation of the inverse FT of the $h_{i j}(k)$. However, within the PY approximation, the $h_{i j}(r)$ 's show discontinuities for $r=d_{i j}$, and trying to compute the inverse FT numerically gives rise to large oscillations (Gibbs oscillations) at the contact value.
To overcome this defect, let us write $h_{i j}(r)$ as

$$
\begin{aligned}
h_{i j}(r) & =h_{i j}(r)+\Delta_{i j} \Theta\left(R_{i j}-r\right)-\Delta_{i j} \Theta\left(R_{i j}-r\right) \\
& =h_{i j}^{*}(r)-\Delta_{i j} \Theta\left(R_{i j}-r\right)
\end{aligned}
$$

where $\Delta_{i j}=g_{i j}\left(d_{i j}^{+}\right)$(the contact value of the RDF) and $\Theta$ is the Heaviside step function. The FT of $h_{i j}$ can be determined from the FT of $\Theta\left(R_{i j}-r\right)$

$$
h_{i j}(q)=h_{i j}^{*}(q)+\Delta_{i j} \hat{\theta}(k)
$$

with

$$
\begin{aligned}
\hat{\theta}(k) & =\mathrm{FT}\left\{\theta\left(R_{i j}-r\right)\right\} \\
& =\frac{4 \pi}{k} \int_{0}^{\infty} \sin (k r) \Theta\left(R_{i j}-r\right) \mathrm{d} r \\
& =\frac{4 \pi}{k^{3}}\left[\sin \left(R_{i j} k\right)-R_{i j} k \cos \left(R_{i j} k\right)\right]
\end{aligned}
$$

and the IFT of $h_{i j}^{*}(q)$, which now shows no discontinuity, can be computed. From $h_{i j}^{*}(r)$, we recover $g_{i j}(r)=h_{i j}(r)+1$.

2. Grundke-Hendersen Correction. The OZ relations were solved using the PY closure for binary hard-sphere mixtures. However, PY hard-sphere distribution functions have two major shortcomings with respect to the values at contact $g_{i j}\left(d_{i j}\right)$ and the slopes $g_{i j}^{\prime}\left(d_{i j}\right)$, both of which are too small. Verlet and Weis gave a procedure to approximately overcome these defects in the pure fluid case, and Grundke and Hendersen generalized this procedure to apply to mixtures ${ }^{77,87}$

$$
\begin{aligned}
& g_{i j}\left(\frac{r}{d_{i j}}, \eta\right)=g_{i j}^{\mathrm{PY}}\left(\frac{r}{d_{i j}^{\prime}}\right)+\Delta g_{i j}(r) \\
& \Delta g_{i j}(r)=\frac{A_{i j}}{r} \exp \left(-b_{i j}\left(r-d_{i j}\right)\right) \times \cos \left(b_{i j}\left(r-d_{i j}\right)\right)
\end{aligned}
$$

with

$$
d_{i j}^{\prime}=d_{i j}\left(1+\frac{\eta}{16}\right)^{3}
$$

The slight change in sphere diameter in the argument of $g^{\mathrm{PY}}(r)$ makes for a small correction in the phase of the oscillations of the distribution function. The purpose of the $A_{i j}$ coefficients is to raise the value of $g(r)$ at contact. Since $\Delta g_{i j}$ must oscillate and the PY solution gives a good approximation for the radial distribution function at large $r$, a trigonometric factor which is damped by $(1 / r) \exp \left(b_{i j}\left(d_{i j}-r\right)\right)$ is included.

According to the MCSL equation of state, the pressure is given by a linear combination of the pressure obtained from the PY integral equation for the RDF following the compressibility and the virial routes, $p^{\mathrm{MCSL}}=(1 / 3) p_{p}^{\mathrm{PY}}+(2 / 3) p_{c}^{\mathrm{PY}}$. The $\mathrm{PY}$ radial distribution function at contact will give, by definition, the $p_{p}^{\mathrm{PY}}$ when used in the pressure equation. Moreover, the scaled particle theory (SPT) for mixtures also reproduces $p_{p}^{\mathrm{PY}}$ via the pressure equation. The contact value of the radial distribution function can then be written as

$$
g_{i j}\left(d_{i j}\right)=\frac{1}{3} g_{i j}^{\mathrm{PY}}\left(d_{i j}\right)+\frac{2}{3} g_{i j}^{\mathrm{SPT}}\left(d_{i j}\right)
$$


This postulate determined the $A_{i j}$ coefficients and forced the distribution functions to give $p^{\mathrm{MCSL}}$ when used in the pressure equation

$$
A_{i j}=\left[\frac{1}{3} g_{i j}^{\mathrm{PY}}(1, \eta)+\frac{2}{3} g_{i j}^{\mathrm{SPT}}(1, \eta)-g_{i j}^{\mathrm{PY}}\left(\frac{d_{i j}}{d_{i j}^{\prime}}, \eta\right)\right] d_{i j}
$$

The pressure consistency can also be generalized to provide the $b_{i j}$ 's. ${ }^{87}$ Lee and Levesque gave the following approximation, ${ }^{88}$ which avoids the numerically involved computation of $\left(\partial \mu_{j}\right) /\left(\partial \rho_{i}\right)$ from the compressibility equation

$$
b_{i j} \approx 24 \frac{A_{i j}}{\eta^{\prime} g_{i j}^{\mathrm{PY}}\left(1, \eta^{\prime}\right) d_{i j}^{2}}
$$

\section{References and Notes}

(1) Dawson, K. A.; Foffi, G.; Sciortino, F.; Tartaglia, P.; Zaccarelli, E. J. Phys.: Condens. Matter 2001, 13, 9113.

(2) Stradner, A.; Sedgwick, H.; Cardinaux, F.; Poon, W. C. K.; Egelhaaf, S. U.; Schurtenbergerpiazza, P. Nature 2004, 432, 492.

(3) Cardinaux, F.; Gibaud, T.; Stradner, A.; Schurtenberger, P. Phys. Rev. Lett. 2007, 99, 118301.

(4) Piazza, R. Curr. Opin. Colloid Interface Sci. 2004, 8, 515.

(5) Likos, C. N. Phys. Rep. 2001, 348, 267.

(6) Louis, A. A. Philos. Trans. R. Soc. London, Ser. A 2001, 359, 939.

(7) Hansen, J.-P.; McDonald, I. R. Theory of Simple Liquids 2nd ed.; Academic Press: London, 1986.

(8) Frenkel, D. Science 2002, 296, 65.

(9) Poon, W. Science 2004, 304, 830.

(10) Anderson, V. J.; Lekkerkerker, H. N. W. Nature 2002, 416, 811.

(11) Zaccarelli, E. J. Phys.: Condens. Matter 2007, 19, 323101.

(12) Foffi, G.; Dawson, K. A.; Buldyrev, S. V.; Sciortino, F.; Zaccarelli, E.; Tartaglia, P. Phys. Rev. E 2002a, 65, 050802(R).

(13) Pellicane, D. C. G.; Caccamo, C. JJ. Phys.: Condens. Matter 2004, $16, \mathrm{~S} 4923$.

(14) Benedek, G. B. Invest. Ophthalmol. Visual Sci. 1997, 38, 1911.

(15) Pitts, L. C. D.; Jose, J.; Lorman, S.; Moss, E.; Varma, S.; Zigler, S.; Zigman, S.; Zuclich, J. Optical Radiation and Visual Health, CRC Press: Boca Raton, FL, 1986.

(16) Benedek, G. B. Appl. Opt. 1971, 10, 459.

(17) Delaye, M.; Tardieu, A. Nature 1983, 302, 415.

(18) Finet, S.; Tardieu, A. J. Cryst. Growth 2001, 232, 40.

(19) Thomson, J. A.; Schurtenberger, P.; Thurston, G. M.; Benedek, G. B. Proc. Natl. Acad. Sci. U.S.A. 1987, 84, 7079.

(20) Broide, M. L.; Berland, C. R.; Pande, J.; Ogun, O. O.; Benedek, G. B. Proc. Natl. Acad. Sci. U.S.A. 1991, 88, 5660.

(21) Malfois, M.; Bonnet, F.; Belloni, L.; Tardieu, A. J. Chem. Phys. 1996, 105,3290

(22) Fine, B. M.; Lomakin, A.; Ogun, O. O.; Benedek, G. B. J. Chem. Phys. 1996, 104, 326.

(23) Lomakin, A.; Asherie, N.; Benedek, G. B. J. Chem. Phys. 1996, $104,1646$.

(24) Asherie, N.; Pande, J.; Lomakin, A.; Ogun, O.; Hanson, S. R.;

Smith, J. B.; Benedek, G. B. Biophys. Chem. 1998, 75, 213.

(25) Zigman, S.; Lerman, S. Nature 1964, 203, 662.

(26) Schurtenberger, P.; Chamberlin, R. A.; Thurston, G. M.; Thomson,

J. A.; Benedek, G. B. Phys. Rev. Lett. 1989, 63, 2064.

(27) Schurtenberger, P.; Chamberlin, R. A.; Thurston, G. M.; Thomson,

J. A.; Benedek, G. B. Phys. Rev. Lett. 1993, 71, 3395.

(28) Thurston, G. M. J. Chem. Phys. 2006, 124, 134909.

(29) Vérétout, F.; Tardieu, A. Eur. Biophys. J. 1989, 17, 61.

(30) Liu, C.; Asherie, N.; Lomakin, A.; Pande, J.; Ogun, O.; Benedek,

G. B. Proc. Natl. Acad. Sci. U.S.A. 1996, 93, 377.

(31) Liu, C.; Pande, J.; Lomakin, A.; Ogun, O.; Benedek, G. B. Invest. Ophthalmol. Visual Sci. 1998, 39, 1609.

(32) Stradner, A.; Foffi, G.; Dorsaz, N.; Thurston, G.; Schurtenberger,

P. Phys. Rev. Lett. 2007, 99, 198103.

(33) Horwitz, J. Proc. Natl. Acad. Sci. U.S.A. 1992, 89, 10449.

(34) Putilina, T.; Skouri-Panet, F.; Prat, K.; Lubsen, N. H.; Tardieu, A.

J. Biol. Chem. 2003, 278, 13747.

(35) Pigaga, V.; Quinlan, R. A. Exp. Cell Res. 2006, 312, 51.

(36) Blundell, T.; Lindley, P.; Miller, L.; Moss, D.; Slingsby, C.; Tickle,

I.; Turnell, B.; Wistow, G. Nature 1981, 289, 771.

(37) Bettelheim, F. A.; Ansari, R.; Cheng, Q.-F.; Zigler, J. S. Biochem. Biophys. Res. Commun. 1999, 261, 292.
(38) Foffi, G.; McCullagh, G. D.; Lawlor, A.; Zaccarelli, E.; Dawson, K. A.; Sciortino, F.; Tartaglia, P.; Pini, D.; Stell, G. Phys. Rev. E 2002, 65, 31407.

(39) Noro, M.; Frenkel, D. J. Chem. Phys. 2000, 113, 2941.

(40) Rapaport, D. C. The Art of Molecular Dynamic Simulation; Cambridge University Press: New York, 1995.

(41) Klein, R. In Neutrons, X-rays and Light: Scattering Methods Applied to Soft Condensed Matter; Zemb, T., ed.; Elsevier Science B.V.: Amsterdam, 2002.

(42) Caccamo, C. Phys. Rep. 1996, 274, 1.

(43) Glatter, O. J. Appl. Crystallogr. 1977, 10, 415.

(44) Guinier, F. G. Small-Angle Sctattering of X-rays; Wiley: New York, 1955.

(45) Pedersen, J. S.; Posselt, D.; Mortensen, K. J. Appl. Crystallogr. 1990, 23, 321.

(46) Stradner, A.; Lobaskin, V.; Schurtenberger, P.; Thurston, G. Structure and Interactions of Lens Proteins in Dilute and Concentrated Solutions. Prog. Colloid Polym. Sci. 2004, 126, 173-177.

(47) Vega, L.; de Miguel, E.; Rull, L. F. J. Chem. Phys. 1992, 96, 2296.

(48) Kumaraswamy, V. S.; Lindley, P. F.; Slingsby, C.; Glover, I. D. Acta Crystallogr., Sect. D 1996, 52, 611.

(49) Najmudin, S.; Nalini, V.; Driessen, H. P.; Slingsby, C.; Blundell,

T. L.; Moss, D. S.; Lindley, P. F. Acta Crystallogr., Sect. D 1993, 49, 223.

(50) Lomakin, A.; Asherie, N.; Benedek, G. B. Proc. Natl. Acad. Sci. U.S.A. 1999, 96, 9465.

(51) Sear, R. P. J. Chem. Phys. 1999, 111, 4800.

(52) DeMichele, C.; Gabrielli, S.; Tartaglia, P.; Sciortino, F. J. Phys. Chem. B 2006, 110, 8064.

(53) Vérétout, F.; Delaye, M.; Tardieu, A. J. Mol. Biol. 1989, 205, 713.

(54) Muschol, M.; Rosenberger, F. J. Chem. Phys. 1997, 107, 1953.

(55) Piazza, R. Curr. Opin. Colloid Interface Sci. 2000, 5, 38.

(56) Bera, S.; Ghosh, S. K. Biophys. Chem. 1998, 70, 147.

(57) Shand-Kovach, I. Electrostatic Properties of Phase-Separating Bovine Lens Proteins. Ph.D. Thesis, Massachusetts Institute of Technology, Cambridge, MA, USA, 1992.

(58) Verwey, E. J. W.; Overbeek, J. T. G. J. Polym. Sci. 1949, 4, 413. (59) Leunissen, M. E.; Christova, C. G.; Hynninen, A.-P.; Royall, C. P.; Campbell, A. I.; Imhof, A.; Dijkstra, M.; van Roij, R.; van Blaaderen, A. Nature 2005, 437, 235.

(60) Zoetekouw, B.; van Roij, R. Phys. Rev. Lett. 2006, 97, 258302.

(61) Chan, D.; Healy, T. W.; White, L. R. J. Chem. Soc., Faraday Trans.

1 1976, 72, 2844.

(62) McQuarrie, D. A. Statistical Mechanics; University Science Books: Sausalito, CA, 1976

(63) Lee, B. P.; Fisher, M. E. Phys. Rev. Lett. 1996, 76, 2906.

(64) Bostrom, M.; Williams, D.; Ninham, B. J. Phys. Chem. B 2002, 106, 7908 .

(65) Kirkwood, J. G.; Shumaker, J. B. Proc. Natl. Acad. Sci. U.S.A. 1952, 38, 855 .

(66) Liu, J.; Wilding, N. B.; Luijten, E. Phys. Rev. Lett. 2006, 97, 115705.

(67) Takemoto, L. J.; Ponce, A. A. Exp. Eye Res. 2006, 83, 793.

(68) Ponce, A.; Sorensen, C.; Takemoto, L. Mol. Vision 2006, 12, 879.

(69) Pini, D.; Tau, M.; Parola, A.; Reatto, L. Phys. Rev. E 2003, 67, 046116.

(70) Scholl-Paschinger, E.; Kahl, G. J. Chem. Phys. 2003, 118, 7414. (71) Gast, A. P.; Russell, W. B.; Hall, C. K. J. Colloid Interface Sci. 1983, 96, 251.

(72) Barker, J. A.; Henderson, D. J. Chem. Phys. 1967, 47, 2856.

(73) Carnahan, N. F.; Starling, K. E. J. Chem. Phys. 1969, 51, 635

(74) Boublík, T. J. Chem. Phys. 1970, 53, 471.

(75) Mansoori, G. A.; Carnahan, N. F.; Starling, K. E.; Leland, J. T. W.

J. Chem. Phys. 1971, 54, 1523.

(76) Lebowitz, J. L. Phys. Rev. A 1964, 133, 895.

(77) Verlet, L.; Weis, J.-J. Phys. Rev. A 1972, 5, 939.

(78) Henderson, D.; Grundke, E. W. J. Chem. Phys. 1975, 63, 601.

(79) Gil-Villegas, A.; Galindo, A.; Whitehead, P. J.; Mills, S. J.; Jackson,

G.; Burgess, A. N. J. Chem. Phys. 1997, 106, 4168.

(80) Paricaud, P. J. Chem. Phys. 2006, 124, 154505.

(81) Tisza, L. Generalized Thermodynamics;MIT: Cambridge, MA, 1966

(82) Ursenbach, C. P.; Patey, G. N. J. Chem. Phys. 1994, 100, 3827.

(83) Schall-Paschinger, E.; Benavides, A. L.; Castaeda-Priego, R. J. Chem. Phys. 2005, 123, 234513.

(84) Biben, T.; Hansen, J.-P. Phys. Rev. Lett. 1991, 66, 2215. 970.

(85) Takemoto, L.; Ponce, A.; Sorensen, C. M. Mol. Vision 2008, 14,

(86) ten Wolde, P. R.; Frenkel, D. Science 1997, 277, 1975.

(87) Grundke, E. W.; Henderson, D. Mol. Phys. 1972, 24, 269.

(88) Lee, L. L.; Levesque, D. Mol. Phys. 1973, 26, 1351. 